\title{
Mixed Equilibrium in a Downsian Model with a Favored Candidate*
}

\author{
Enriqueta Aragonés ${ }^{\dagger}$ \\ Universitat Pompeu Fabra
}

\author{
Thomas R. Palfrey \\ California Institute of Technology
}

October 13, 2000

\begin{abstract}
This paper examines competition in the standard one-dimensional Downsian model of two-candidate elections, but where one candidate $(A)$ enjoys an advantage over the other candidate $(D)$. Voters' preferences are Euclidean, but any voter will vote for candidate $A$ over candidate $D$ unless $D$ is closer to her ideal point by some fixed distance $\delta$. The location of the median voter's ideal point is uncertain, and its distribution is commonly known by both candidates. The candidates simultaneously choose locations to maximize the probability of victory. Pure strategy equilibria often fails to exist in this model, except under special conditions about $\delta$ and the distribution of the median ideal point. We solve for the essentially unique symmetric mixed equilibrium, show that candidate $A$ adopts more moderate policies than candidate $D$, and obtain some comparative statics results about the probability of victory and the expected
\end{abstract}

*Aragones acknowledges financial support by the Generalitat de Catalunya Grant number 1999SGR 00157 and the University Pompeu Fabra Grant number COFREA99.003 and the hospitality of CBRSS at Harvard University. Palfrey acknowledges financial support from the National Science Foundation, grant number SBR-9631627. We are grateful to Tim Groseclose and participants at the Wallis Conference on Candidate Entry, Exit, and Positioning, University of Rochester, June 2000, for their comments.

${ }^{\dagger}$ Departament d'Economia, Universitat Pompeu Fabra, Carrer Ramon Trias Fargas 25-27, 08005 Barcelona, Spain. aragones@upf.es

$\ddagger$ (corresponding author) Division of Humanities and Social Sciences, Mail Code 228-77, California Institute of Technology, Pasadena, CA 91125. trp@hss.caltech.edu Fax:(626)432-1726 
distance between the two candidates' policies. We find that both players' equilibrium strategies converge to the expected median voter as $A^{\prime} s$ advantage shrinks to zero.

Key words: spatial competition; mixed strategies; candidate quality

\section{Introduction}

Often the media tells us about Candidate So-and-so's charisma, hand-shaking skill, great speech delivery, or lack thereof. Yet only rarely do these features find leading roles in the simple spatial models that political scientists have embraced as the tool of choice to study candidate competition in mass elections. This paper takes the simplest non-trivial extension of the standard Downsian model in this direction, and explores the implications for the equilibrium positioning of candidates. With this seemingly trivial addition of realism into the model things seem to change very dramatically. Pure strategy equilibria fail to exist even in a single dimension where voters have single-peaked preferences. Candidates diverge, and this divergence occurs in predictable ways. Candidates with charisma end up reinforcing their advantage by adopting relatively more centrist platforms on average, while the ugly, clumsy, and inarticulate flounder on the periphery of the policy space.

The implications of our model are actually more general than simply an investigation of the effects of charisma, or other "exogenous" candidate characteristics. The results apply for any particular non-policy advantage one candidate has over another, which is valued by all voters. Thus, endogenous political phenomena such as office holding experience, incumbent performance, constituency service, and advertising (campaign expenditures) can also generate similar effects. Because these advantages or disadvantages can arise for either endogenous or exogenous reasons, we lump all of them together and simply view these effects as "image". In addition to the candidate-specific image dimension, there are also broader "valence" issues (Stokes, 1963, pp. 170-4), such as economic performance (Kiewiet 1983) and military success, that are irreversibly linked to a candidate through his or her party's past and current performance. There is ample evidence that such issues are very important in elections, and such factors need to be incorporated into the standard models. To wit:

"It will not do simply to exclude valence issues from the discussion of party competition. The people's choice too often depends upon them. At least in American presidential elections of the past generation it is remarkable how many valence issues have held the center stage." (Stokes, 1963, p. 171) 
More recently, Popkin et al. (1976) come to a similar conclusion about the 1972 U.S. election: "[The voter] cares less about which candidate is the closest to his ideal position on issues for which he has information and preferences, but cares most about which candidate can deliver the most" (p. 793). Since we find here that asymmetries along the image dimension or divergent voter perceptions of candidate competence on valence issues can generate significant electoral effects in equilibrium, this may help explain why empirical studies in political science often find these kinds of effects play important roles in campaigns, candidate entry decisions (Banks and Kiewiet 1989), and incumbent longevity (Kiewiet and Zeng 1993).

We also obtain a continuity result suggesting that the standard Downsian model may be a good approximation when these valence dimensions play a minor role in the election. In particular, in our model, as the advantage to one candidate shrinks to zero, each of the candidate's

There are several recent papers that have begun to investigate the theoretical properties of equilibrium in the presence of candidate image effects. The closest are Ansolabehere and Snyder (2000) and Groseclose (1999). ${ }^{1}$ Those papers investigate different variations of the model we study in this paper, and they focus only on pure strategy equilibria. Ansolabehere and Snyder (2000) focus on candidates that maximize the probability of winning in a world of certainty. They find that, when a pure strategy equilibrium exists, the advantaged candidate locates centrally, and there is no restriction on the location of the disadvantaged candidate (who always loses). In Groseclose, candidates have policy preferences of their own, which are sufficiently weighted in their objective function to guarantee pure strategy equilibria. Policy preferences of sufficient magnitude can overcome the problem of nonexistence of pure strategy equilibrium (Groseclose 1999). If two candidates maximize expected vote (or probability of winning, when the median voter's location is random) and are purely office-motivated, then pure strategy equilibrium fails to exist except in uninteresting boundary cases, such as when there is no uncertainty. ${ }^{2}$ This result is indeed very general, covering rather arbitrary policy spaces. The intuition is simple. The advantaged candidate wins all the votes if he exactly copies the location of the disadvantaged candidate. Thus, the disadvantaged candidate must mix in order not to be predictable. However, in order for mixing to be optimal for the disadvantaged candidate, the advantaged candidate also must be mixing.

\footnotetext{
${ }^{1}$ These papers were brought to our attention after we had obtained the main results presented in this paper.

${ }^{2}$ Indeed, this has been known for a long time, and Groseclose (1999) quite correctly refers to it as a folk theorem.
} 
The nonexistence of pure strategy equilibrium, even with arbitrarily small advantagees for one candidate suggests that the standard Downsian model may be a knife-edge case. That is, any small amount of asymmetry between the candidates along the valence dimension could invalidate the standard results. Groseclose (1999) raises exactly such a concern. However, we show that this knife-egde feature is illusive. In spite of the loss of pure strategy equilibria, the distribution of strategies in the mixed equilibrium converges to a degenerate distribution - i.e.,the Downsian pure strategy equilibrium - as the advantage becomes very small, and the two candidates win with equal probability. Thus, our model produces a continuity result that the standard Downsian model is a good approximation when these valence dimensions play a minor role in the election.

Several other papers have examined formal models of candidate equilibria where there are asymmetries between the candidates. Bernhardt and Ingberman (1985) and Berger, Munger, and Potthoff (2000) look at incumbency advantages, and explore equilibria when candidates locate sequentially. Londregan and Romer (1993) also look at competition with incumbency effects. Wittman (2000) adopts a sequential location approach, in a model that looks at the role of pressure groups. Sequential approaches like these avoid the nonexistence problem of simultaneous location choice, but leave open the question of what is the correct sequential model, which can be problematic since results typically depend on order of moves.

None of the above papers investigates the properties of the mixed strategy equilibria of the simultaneous location game. That is what we do in this paper. In section 2 we present the basic model, where the policy space is a finite grid of points on the $[0,1]$ interval, voters have Euclidean preferences, the location of the median voter's ideal point is uncertain, and candidates choose policies to maximize probability of winning. In section 3 we solve for closed form solutions of mixed strategy equilibrium when the size of the advantage enjoyed by one candidate is relatively small. It turns out that the solutions are slightly different depending on whether the policy space consists of an even or an odd number points, so we analyze the two cases separately. The basic techniques in the two cases are similar, so we only include the analysis of the even case in the body of the paper. The odd case is treated in an appendix, where we also prove that the two solutions converge when the policy space grid becomes fine. In section 4, we analyze the properties of the equilibrium found in the previous section for large $n$. We compare the expected payoffs of the two candidates, and look at limiting cases, in which the advantage is arbitrarily small and the policy space grid approaches a continuum. We find that the advantaged candidate always has a higher expected payoff in equilibrium, but this equilibrium advantage shrinks to zero in the limit. In section 5 we explore three different extensions of the model: non-uniform 
distributions of the median voter's ideal point, larger values of the advantage, and existence of a mixed strategy equilibrium in the case of continuous locations. We conclude in section 6 .

\section{The Model}

The policy space, $\wp$, is the set of $n$ points on the [0,1] interval, $x_{i}=\frac{i-1}{n-1}, i=1,2, \ldots, n$. There are two candidates, $A$ and $D$, who are referred to as the advantaged candidate and the disadvantaged candidate, respectively. Each candidate's objective is to maximize his probability of winning the election. Each voter has a utility function, with two components, a policy component, and a candidate image component. ${ }^{3}$ The policy component is characterized by an ideal point in the policy space $\wp$, with utility of alternatives in the policy space a strictly decreasing function of the Euclidean distance between the ideal point and the location of the policy, symmetric around the ideal point. We assume there exists a unique median location, denoted by $x_{m}$. Candidates do not know $x_{m}$, but share a common prior belief about it. This commonly shared belief is represented by a probability distribution over $\wp$, and is denoted by a vector of probabilities, $\left(\rho_{1}, \ldots, \rho_{n}\right)$, where $\rho_{i} \geq 0, i=1,2, \ldots, n$ and $\rho_{1}+\ldots+\rho_{n}=1$. We denote by $m$ the median of the distribution $\rho$. The image component is captured by an additive constant to the utility a voter gets if $A$ wins the election. That is, the utility that a voter with ideal point $x_{i}$ obtains if $A$ wins the election is $U_{i}\left(x_{A}\right)=\delta-\left|x_{i}-x_{A}\right|$ and his utility if candidate $D$ wins is $U_{i}\left(x_{D}\right)=-\left|x_{i}-x_{D}\right|$, where candidates' policy positions are denoted by $x_{A}$ and $x_{B}$, the size of $A$ 's advantage is $\delta \geq 0 .{ }^{4}$

The game takes place in two stages. In the first stage, candidates simultaneously choose positions in $\wp$. In the second stage, each voter votes for the candidate whose election would give him the higher utility. In case of indifference, a voter is assumed to vote for each candidate with probability equal to $1 / 2 .^{5}$

Since the behavior of the voters is unambiguous in this model, we define an equilibrium of the game only in terms of the location strategies of the two candidates in the first round. A pure strategy equilibrium is a pair of candidate locations, $\left(x_{A}, x_{D}\right)$ such that both candidates are maximizing the probability of winning, given the choices of

\footnotetext{
${ }^{3}$ There could be either a finite number of voters or a continuum.

${ }^{4}$ Two natural generalization of this model would be: (1) to allow different candidates to have different beliefs about $x$; or (2) to allow different voters to have different image terms. Here we assume homogeneity among voters and among candidates.

${ }^{5}$ All our results would still hold if indifferent voters vote with any arbitrary probability for each candidate.
} 
the other candidate. We denote by $\pi_{A}\left(x_{A}, x_{D}\right)$ and $\pi_{D}\left(x_{A}, x_{D}\right)$ the probability of winning for candidate $A$ and for candidate $D$, respectively, as a function of $\left(x_{A}, x_{D}\right) .{ }^{6}$ A mixed strategy equilibrium is a pair of probability distributions over $\wp,\left(\sigma^{A}, \sigma^{D}\right)$, such that there is no mixed strategy for $A$ that guarantees higher probability of winning than $\sigma^{A}$, given $\sigma^{D}$ and there is no mixed strategy for $D$ that guarantees higher probability of winning than $\sigma^{D}$, given $\sigma^{A}$.

\section{Derivation of Mixed equilibrium}

For the rest of this section, we consider only small values of $\delta$, such that $0 \leq \delta<\frac{1}{n-1}$. This simplifies the derivation of equilibrium considerably. In the last section, we consider what happens for larger values of $\delta$. When $\delta=0$, neither candidate has an advantage, and we are in the standard Downsian world. In this case, when the distribution $\rho$ has a unique median, the two candidates both locate at the median $m$. The two candidates each win with probability .5. Otherwise, there are multiple equilibria because there is no unique median location. All of these equilibria involve the two candidates mixing between the two median locations. As is evident from this, the even and odd cases will have to be treated separately for the case of $\delta>0$. When $0<\delta<\frac{1}{n-1}$, candidate $A$ wins if and only if the ideal point of the median voter is at least as close to $A$ as to $B$. If $B$ is strictly closer to the median voter's ideal point than is $A$, then $B$ wins.

First we state a version of a simple result that has also been established elsewhere. ${ }^{7}$ There is never an equilibrium in pure strategies.

Proposition 1: If $0<\delta<\frac{1}{n-1}$ and $n>1$, then there does not exist a pure strategy equilibrium.

Proof: Suppose that $\left(x_{A}, x_{D}\right)$ were an equilibrium. If $x_{A}=x_{D}$, then $\pi_{D}\left(x_{A}, x_{D}\right)=$ 0 . Since $n>1$, there exists some $x \neq x_{D}$, and $\pi_{D}\left(x_{A}, x\right) \geq \frac{1}{n}>\pi_{D}\left(x_{A}, x_{D}\right)$, so it must be that $x_{A} \neq x_{D}$. But if $x_{A} \neq x_{D}$, then $\pi_{A}\left(x_{A}, x_{D}\right) \leq \frac{n-1}{n}<1=\pi_{D}\left(x_{D}, x_{D}\right)$, so $\left(x_{A}, x_{D}\right)$ is not an equilibrium.

The intuition is simple. If the disadvantaged candidate's location is perfectly predictable, the advantaged candidate can copy that strategy and win for sure. There-

\footnotetext{
${ }^{6}$ This model is formally equivalent to one in which there are $n$ voters with Euclidean preferences, whose ideal points are equally spaced along the unit interval, there is no uncertainty, and candidates maximize expected vote.

${ }^{7}$ Versions of this theorem, with proofs, can be found in Groseclose (1999) and Berger, Munger, and Potthoff (1999).
} 
fore, (at least) the disadvantaged candidate must be mixing. The result extends easily to larger values of $\delta$, and is true unless $\delta$ is sufficiently large that $A$ can locate at the median and guarantee a payoff of 1 . In general, if $\delta<\frac{n}{2}-1$, then there will be no pure strategy equilibrium. In what follows, we limit attention to uniform distribution of the medians, and to strategies that have a particular symmetry property. Specifically, each of the mixed strategy distributions of the candidates are symmetric around $\frac{1}{2}$. Formally:

Definition 1: A strategy for candidate $j$ is symmetric if $\sigma_{j i}=\sigma_{j, n-i+1}$.

Also, at least in this section, we will limit attention to equilibria in which there are "no gaps." That is, the support of each candidate's mixed strategy is an interval. Formally:

Definition 2: A strategy for candidate $j$ has no gaps if there exist integers $i, k$ such that $0 \leq i \leq k \leq n$ and $\sigma_{j t}>0$ if and only if $i \leq t \leq k$.

We will show below, by construction, the existence of symmetric equilibria with no gaps, for this case of small $\delta$. We have not been able to rule out the possibility that there are mixed equilibria that are asymmetric and/or have gaps.

In the remainder of the paper, we assume there are an even number of locations and $\rho$ is uniform, unless specifically stated otherwise. The analysis for the odd case is given in appendix A. In this section we derive the symmetric equilibrium strategies with no gaps for a fixed even value of $n$, greater than or equal to 8 . The equilibrium mixed strategies for $n=4$ and $n=6$ are described in section 3.4 as examples.

\subsection{Candidate $A$ 's payoffs for each strategy}

Given any mixed strategy, $\sigma^{D}=\left(\sigma_{1}^{D}, \ldots, \sigma_{n}^{D}\right)$ for candidate $D$, we denote by $\pi_{A}\left(x_{i}, \sigma^{D}\right)$ the probability of winning for candidate $A$ if $A$ chooses location $x_{i}, i=1, \ldots, n$, where $x_{i}=\frac{i-1}{n-1}$. These expressions are written below: ${ }^{8}$

In general the payoffs of candidate $A$ for a given strategy $i$ can be written as: ${ }^{9}$

$$
n \pi_{A}\left(x_{i}, \sigma^{D}\right)=\sum_{j=1}^{\left[\frac{i-1}{2}\right]}(n-i+j+1) \sigma_{i-2 j-1}^{D}+\sum_{j=1}^{\left[\frac{i-1}{2}\right]}(n-i+j+1) \sigma_{i-2 j}^{D}
$$

\footnotetext{
${ }^{8}$ For the case of $n<8$, some minor modification is needed.

${ }^{9}$ For some values of $i$ in the summation expressions below, subscripts are out of range (e.g., $i=1$ ), in which case those terms are set equal to 0 . The " $[x]$ " notation denotes the greatest integer less than or equal to $x$.
} 


$$
+(n-i+1) \sigma_{i-1}^{D}+n \sigma_{i}^{D}+i \sigma_{i+1}^{D}+\sum_{j=1}^{\left[\frac{n-i}{2}\right]}(i+j) \sigma_{i+2 j}^{D}+\sum_{j=1}^{\left[\frac{n-i}{2}\right]}(i+j) \sigma_{i+2 j+1}^{D}
$$

For example, for $i=3$, this reduces to:

$n \pi_{A}\left(x_{3}, \sigma^{D}\right)=(n-1) \sigma_{1}^{D}+(n-2) \sigma_{2}^{D}+n \sigma_{3}^{D}+3 \sigma_{4}^{D}+4 \sigma_{5}^{D}+\ldots+\left(\frac{n}{2}+1\right) \sigma_{n-1}^{D}+\left(\frac{n}{2}+1\right) \sigma_{n}^{D}$

Symmetric equilibria with no gaps are solved by (1) equating the payoffs of adjacent locations, and then (2) finding one of the endpoints of the support. If adjacent locations $x_{i}$ and $x_{i+1}$ are used with positive probability by candidate $A$ in equilibrium, then their expected payoffs (probability of winning) must be equal. For example, if locations 1 and 2 are both in the support of $A$ 's mixed strategy, then it must be that:

$$
n \pi_{A}\left(x_{1}, \sigma^{D}\right)=n \pi_{A}\left(x_{2}, \sigma^{D}\right) \Longrightarrow \sigma_{1}^{D}+(1-n) \sigma_{2}^{D}-\sum_{i=2}^{\frac{n}{2}} \sigma_{2 i}^{D}=0
$$

If we denote by $k_{A}^{*}$ the first location in the support of $A^{\prime} s$ strategy, then we have a collection of $n-2 k_{A}^{*}+1$ equations of the form:

$$
\begin{aligned}
k \sigma_{k}^{D}+\sum_{i=1}^{\left[\frac{k-1}{2}\right]} \sigma_{k-2 i}^{D} & =(n-k) \sigma_{k+1}^{D}+\sum_{i=1}^{\left[\frac{n-k-1}{2}\right]} \sigma_{k+2 i+1}^{D} \\
\text { for } k_{A}^{*} & \leq k \leq n-k_{A}^{*}
\end{aligned}
$$

where the partial sums continue up to the point where the subscripts become either less than 1 or greater than $n .^{10}$

If we further assume symmetry, that is, $\sigma_{k}^{D}=\sigma_{n-k+1}^{D}$ we obtain the following simple system of equations that can be solved to obtain the mixing probabilities of $D$ :

$$
(k-1) \sigma_{k}^{D}=(n-k) \sigma_{k+1}^{D}+\sum_{i=k+2}^{\frac{n}{2}} \sigma_{i}^{D} \text { for } k_{A}^{*} \leq k<\frac{n}{2}
$$

\subsection{Candidate $D$ 's payoffs for each strategy}

We derive the equilibrium conditions for candidate $D$ in a similar fashion. Given any mixed strategy, $\sigma^{A}=\left(\sigma_{1}^{A}, \ldots, \sigma_{n}^{A}\right)$ for candidate $A$, we denote by $\pi_{D}\left(x_{i}, \sigma^{A}\right)$ the

\footnotetext{
${ }^{10}$ Note that for low values of $n$ this formula needs to be adjusted at the bounds.
} 
probability of winning for candidate $D$ if $D$ chooses location $x_{i}, i=1, \ldots, n$, where $x_{i}=\frac{i-1}{n}$. So, the payoffs of candidate $D$ for a given strategy $i$ can be written as: ${ }^{11}$

$$
\begin{aligned}
& n \pi_{D}\left(x_{i}, \sigma^{A}\right)=\sum_{j=1}^{\left[\frac{i}{2}\right]}(n-i+j) \sigma_{i-2 j+1}^{A}+\sum_{j=1}^{\left[\frac{i}{2}\right]}(n-i+j) \sigma_{i-2 j}^{A} \\
& +\sum_{j=0}^{\left[\frac{n-i}{2}\right]}(i+j) \sigma_{i+2 j+1}^{A}+\sum_{j=0}^{\left[\frac{n-i}{2}\right]}(i+j) \sigma_{i+2 j+2}^{A}
\end{aligned}
$$

Equating candidate $D^{\prime} s$ payoffs on the support of $D^{\prime} s$ strategy, we obtain a collection of $n-2 k_{D}^{*}+1$ equations of the form:

$$
\begin{aligned}
k \sigma_{k+1}^{A}+\sum_{i=1}^{\left[\frac{k}{2}\right]} \sigma_{k-2 i+1}^{A} & =(n-k) \sigma_{k}^{A}+\sum_{i=1}^{\left[\frac{n-k}{2}\right]} \sigma_{k+2 i}^{A} \\
\text { for } k_{D}^{*} & \leq k \leq n-k_{D}^{*}
\end{aligned}
$$

where the partial sums continue up to the point where the subscripts become either less than 1 or greater than $n$.

Imposing symmetry, $\sigma_{k}^{A}=\sigma_{n-k+1}^{A}$ we obtain the mixing probabilities of $A$ :

$$
(k-1) \sigma_{k+1}^{A}=(n-k) \sigma_{k}^{A}+\sum_{i=k+2}^{\frac{n}{2}} \sigma_{i}^{A} \quad \text { for } k_{D}^{*} \leq k<\frac{n}{2}
$$

\subsection{Equilibrium in symmetric strategies}

¿From the set of equations (1) we obtain the first proposition about the equilibrium strategy of candidate $D$, provided $D$ 's mixing probabilities are symmetric around $\frac{1}{2}$, and there are no "gaps" in candidate $A^{\prime} s$ strategy.

Proposition 2: In any equilibrium with symmetric strategies and no gaps, $\sigma_{k}^{D} \geqslant$ $\sigma_{k+1}^{D}$ for $1 \leqslant k<\frac{n}{2}$ and $\sigma_{k}^{D} \leqslant \sigma_{k+1}^{D}$ for $\frac{n}{2} \leqslant k \leqslant n-1$.

Proof: If $k \leqslant \frac{n+1}{2}$ then $n-k \geqslant k-1$. From expression (1), this immediately implies that $\sigma_{k}^{D} \geqslant \sigma_{k+1}^{D}$ for $1 \leqslant k<\frac{n}{2}$

Proposition 3: In any equilibrium with symmetric strategies and no gaps, $\sigma_{k}^{A} \leqslant$ $\sigma_{k+1}^{A}$ for $1 \leqslant k<\frac{n}{2}$ and $\sigma_{k}^{A} \geqslant \sigma_{k+1}^{A}$ for $\frac{n}{2} \leqslant k \leqslant n-1$.

\footnotetext{
${ }^{11}$ For some values of $i$ in the summation expressions below, subscripts are out of range (e.g., $\left.i=1\right)$, in which case those terms are set equal to 0.
} 
Proof: If $k \leqslant \frac{n+1}{2}$ then $n-k \geqslant k-1$. From expression (2), this immediately implies that $\sigma_{k}^{A} \leq \sigma_{k+1}^{A}$ for $1 \leqslant k<\frac{n}{2}$.

That is, the disadvantaged candidate's mixing distribution is $U$-shaped, with the least probability weight in the center. In contrast, candidate $A$ 's mixing distribution places monotonically decreasing weight on strategies that are further from the median. Intuitively, the disadvantaged candidate must differentiate his policy from the advantaged candidate. Completing the picture, the advantaged candidate locating in the center of the policy space (in a probabilistic sense), effectively "forces" the disadvantaged candidate to tend to adopt more extreme positions (again, in a probabilistic sense).

Proposition 4: In any equilibrium with symmetric strategies and no gaps, $\sigma_{k}^{A}>0$ only if $\frac{n}{4} \leq k \leq \frac{3 n}{4}$ and $\sigma_{k}^{D}>0$ only if $\frac{n}{4} \leq k \leq \frac{3 n}{4}$.

Proof: Consider the set of equations (2):

$$
(k-1) \sigma_{k+1}^{A}=(n-k) \sigma_{k}^{A}+\sum_{i=2}^{\frac{n}{2}} \sigma_{k+i}^{A} \quad \text { for } 1 \leqslant k<\frac{n}{2}
$$

If $k \leqslant \frac{n}{4}$ then $k-1 \leqslant \frac{n}{2}-k-1:$ in this case we have that the number of $p$ 's in the RHS larger than or equal to $p_{k+1}$ (considering that $\sigma_{k}^{A}<\sigma_{k+1}^{A}$ for $k<\frac{n}{2}$ ) is larger than the number of $\sigma_{k+1}^{A}$ in the LHS. So these equations cannot be satisfied by any $\sigma^{A}>0$. Therefore, we must have $\sigma_{k}^{D}=0$ for $k \leqslant \frac{n}{4}$, and because of symmetry: $\sigma_{k}^{D}=0$ for $k \geqslant \frac{3 n}{4}$. Now consider equation (1). If $k \leqslant \frac{n}{4}$ the LHS is equal to zero and the RHS is positive, so they cannot hold either. Therefore, we must have $\sigma_{k}^{A}=0$ for $k \leqslant \frac{n}{4}$, and because of symmetry: $\sigma_{k}^{A}=0$ for $k \geqslant \frac{3 n}{4}$.

A final proposition concerns the endpoints of the supports of the mixed strategies, $k_{A}^{*}$ and $k_{D}^{*}$. The main point is that these two endpoints must be very close to each other.

Proposition 5: In any equilibrium with symmetric strategies and no gaps, $\sigma_{k}^{A}>0$ only if $\sigma_{k}^{D}>0$, and hence $k_{D}^{*} \leq k_{A}^{*}$. Furthermore, $k_{A}^{*} \leq k_{D}^{*}+1$, so $k_{D}^{*} \leq k_{A}^{*} \leq k_{D}^{*}+1$.

Proof: If there are no gaps, clearly the support of $A$ cannot strictly contain the support of $D$. A strategy for $A$ that assigns positive probability to a location less than $k_{D}^{*}$ can be improved on by a strategy for $A$ that moves all that probability to location $k_{D}^{*}$, but is the same in all other respects. This yields strictly higher expected payoffs to $A$ since, by assumption, $D$ is placing some probability on $k_{D}^{*}$. Similarly, a 
strategy for $D$ that assigns positive probability to a location less than $k_{A}^{*}-1$ can be improved on by a strategy for $D$ that moves all that probability to location $k_{A}^{*}-1$, but is the same in all other respects. This yields strictly higher expected payoffs to $D$ since, by assumption, $A$ is placing some probability on $k_{A}^{*}$. We cannot rule out $D$ placing positive probability on $k_{A}^{*}-1$.

\subsubsection{Recursive derivation of equilibrium}

If we first consider the equations determining the $A$ mixing probabilities (from the $D$ indifference equations) in order to find the mixed strategy equilibrium, we have: ${ }^{12}$

$$
(n-k) \sigma_{k}^{A}+\sum_{i=2}^{\frac{n}{2}} \sigma_{k+i}^{A}=(k-1) \sigma_{k+1}^{A} \text { for } \frac{n}{4}<k<\frac{n}{2}
$$

These equations can be solved recursively to give:

$$
\begin{aligned}
\sigma_{\frac{n}{2}-1}^{A} & =\frac{\frac{n}{2}-2}{\frac{n}{2}+1} \sigma_{\frac{n}{2}}^{A} \\
\sigma_{\frac{n}{2}-2}^{A} & =\frac{\left(\frac{n}{2}-3\right) \frac{\frac{n}{2}-2}{\frac{n}{2}+1}-1}{\frac{n}{2}+2} \sigma_{\frac{n}{2}}^{A} \\
\sigma_{\frac{n}{2}-3}^{A} & =\frac{\left(\frac{n}{2}-4\right) \frac{\left(\frac{n}{2}-3\right) \frac{\frac{n}{2}-2}{\frac{2}{2}+1}-1}{\frac{n}{2}+2}-\frac{\frac{n}{2}-2}{\frac{n}{2}+1}-1}{\frac{n}{2}+3} \sigma_{\frac{n}{2}}^{A}
\end{aligned}
$$

and so forth.

Similarly, if we consider the equations determining the $D$ mixing probabilities (from the $A$ indifference equations), we have

$$
(k-1) \sigma_{k}^{D}=(n-k) \sigma_{k+1}^{D}+\sum_{i=2}^{\frac{n}{2}} \sigma_{k+i}^{D} \quad \text { for } \quad\left[\frac{n}{4}\right]<k<\frac{n}{2}
$$

\footnotetext{
${ }^{12}$ Of course, not all of these equations will necessarily hold, since we may have $p_{k}=0$ or $q_{k}=0$ for some $k$ between $\frac{n}{4}$ and $\frac{3 n}{4}$. Part of the solution below will involve finding the minimum value of $k$.
} 
These are solved recursively in a similar fashion as above, to get:

$$
\begin{aligned}
\sigma_{\frac{n}{2}-1}^{D} & =\frac{\frac{n}{2}+1}{\frac{n}{2}-2} \sigma_{\frac{n}{2}}^{D} \\
\sigma_{\frac{n}{2}-2}^{D} & =\frac{\left(\frac{n}{2}+2\right)\left(\frac{\frac{n}{2}+1}{\frac{n}{2}-2}\right)+1}{\frac{n}{2}-3} \sigma_{\frac{n}{2}}^{D} \\
\sigma_{\frac{n}{2}-3}^{D} & =\frac{\left(\frac{n}{2}+3\right) \frac{\left(\frac{n}{2}+2\right)\left(\frac{n}{\frac{2}{n}+1}\right)+1}{\frac{n}{2}-3}+\left(\frac{\frac{n}{2}+1}{\frac{n}{2}-2}\right)+1}{\frac{n}{2}-4} \sigma_{\frac{n}{2}}^{D}
\end{aligned}
$$

and so forth.

Since $\sigma^{A}$ and $\sigma^{D}$ are probability distributions, we must have:

$$
\begin{aligned}
\sum_{\left[\frac{n}{4}\right]<k \leqslant \frac{n}{2}} \sigma_{k}^{A} & =\frac{1}{2} \\
\sum_{\left[\frac{n}{4}\right]<k \leqslant \frac{n}{2}} \sigma_{k}^{D} & =\frac{1}{2} \\
\sigma_{k}^{A} & \geq 0, k=1,2, \ldots, n \\
\sigma_{k}^{D} & \geq 0, k=1,2, \ldots, n
\end{aligned}
$$

Therefore, we can solve the two recursive systems explicitly to get:

$$
\sigma_{\frac{n}{2}}^{A}=\frac{1}{2\left(\sum_{0 \leq j \leq j_{A}^{*}} S_{j}^{A}(n)\right)}
$$

and $\sigma_{\frac{n}{2}-j}^{A}=S_{j}^{A}(n) \sigma_{\frac{n}{2}}^{A}$ where:

$$
\begin{aligned}
S_{0}^{A} & =1 \\
S_{1}^{A}(n) & =\frac{\frac{n}{2}-2}{\frac{n}{2}+1}
\end{aligned}
$$




$$
S_{j}^{A}(n)=\frac{\left(\frac{n}{2}-j\right) S_{j-1}^{A}(n)-\sum_{i=0}^{j-1} S_{i}^{A}(n)}{\frac{n}{2}+j}
$$

As $j$ increases $S_{j}^{A}(n)$ may become negative. Let $j^{*}$ denote the largest $j$ for which $S_{j}^{A}(n)$ is positive. It determines the extreme point of the support of $\sigma^{A}$, denoted by $k^{*}$ which is given by: $k^{*}=\frac{n}{2}-j_{A}^{*}$ where $j^{*}=\max \left\{j \geq 1 \mid\left(\frac{n}{2}-j\right) S_{j-1}^{A}(n) \geq\right.$ $\left.1+S_{1}^{A}(n)+S_{2}^{A}(n)+\ldots+S_{j-1}^{A}(n)\right\}$.

A similar solution can be worked out for the $\sigma^{D}$ 's,

$$
\sigma_{\frac{n}{2}}^{D}=\frac{1}{2\left(\sum_{0 \leq i \leq j_{D}^{*}} S_{i}^{D}(n)\right)}
$$

and $\sigma_{\frac{n}{2}-j}^{D}=S_{j}^{D}(n) \sigma_{\frac{n}{2}}^{D}$ where

$$
\begin{aligned}
S_{0}^{D} & =1 \\
S_{1}^{D}(n) & =\frac{\frac{n}{2}+1}{\frac{n}{2}-2} \\
S_{j}^{D}(n) & =\frac{\left(\frac{n}{2}+j\right) S_{j-1}^{D}(n)+\sum_{i=0}^{j-2} S_{i}^{D}(n)}{\frac{n}{2}-j-1}
\end{aligned}
$$

From these recursive equations, we can derive a symmetric equilibrium equilibrium with no gaps. There is always exactly one equliibrium in which the two candidates mix over the same support. Sometimes there is a second equilibrium, which is the same for A, but D mixes over a slightly wider support that includes one additional position to the right and one additional position to the left. There are no other symmetric equilibria with no gaps.

Theorem 1: There is a unique symmetric, no-gap equilibrium mixed strategy for player $A, \bar{\sigma}^{A}$,.with support $\overline{\bar{\Sigma}}_{n}=\left\{x_{\frac{n}{2}-j^{*}}, x_{\frac{n}{2}-j^{*}+1}, \ldots, x_{\frac{n}{2}}, x_{\frac{n}{2}+1}, \ldots, x_{\frac{n}{2}+j^{*}}, x_{\frac{n}{2}+j^{*}+1}\right\}$. If $S_{j^{*}+1}^{A}(n)<0$, then Player $D$ has a unique symmetric, no-gap equilibrium mixed strategy, $\bar{\sigma}^{D}$, with the same support as $\sigma^{A}$. If $S_{j^{*}+1}^{A}(n)=0$, then, in addition to $\bar{\sigma}^{D}$, player $D$ can also have symmetric, no-gap equilibrium mixed strategies on the support $\left\{x_{\frac{n}{2}-j^{*}-1}, x_{\frac{n}{2}-j^{*}}, x_{\frac{n}{2}-j^{*}+1}, \ldots, x_{\frac{n}{2}}, x_{\frac{n}{2}+1}, \ldots, x_{\frac{n}{2}+j^{*}}, x_{\frac{n}{2}+j^{*}+1}, x_{\frac{n}{2}+j^{*}+2}\right\}$. There are 
no other symmetric, no-gap equilibria.

Proof: Consider equation (2)

$$
(n-k) \sigma_{k}^{A}++\sum_{i=2}^{\frac{n}{2}} \sigma_{k+i}^{A}=(k-1) \sigma_{k+1}^{A} \quad \text { for } \frac{n}{4}<k<\frac{n}{2}
$$

Notice that for all $\frac{n}{4}<k<\frac{n}{2}$ we can write $\sigma_{k}^{A}$ as a function of $\sigma_{i}^{A}$ with $k<i \leq \frac{n}{2}$, that is

$$
\sigma_{k}^{A}=\frac{(k-1) \sigma_{k+1}^{A}-\sum_{i=2}^{\frac{n}{2}} \sigma_{k+i}^{A}}{(n-k)} .
$$

In particular we will have that $\sigma_{\frac{n}{2}-1}^{A}$ is a function of $\sigma_{\frac{n}{2}}^{A} ; \sigma_{\frac{n}{2}-2}^{A}$ is a function of $\sigma_{\frac{n}{2}-1}^{A}$ and $\sigma_{\frac{n}{2}}^{A}$. Substituting $\sigma_{\frac{n}{2}-1}^{A}$ from the previous result we can also express $\sigma_{\frac{n}{2}-2}^{A}$ as a function of $\sigma_{\frac{n}{2}}^{A}$. Following this approach for all $1 \leq j<\frac{n}{2}$ we can write each $\sigma_{\frac{n}{2}-j}^{A}$ as a linear function of $\sigma_{\frac{n}{2}}^{A}$, that is, $\sigma_{\frac{n}{2}-j}^{A}=S_{j}^{A}(n) \sigma_{\frac{n}{2}}^{A}$ with coefficients:

$$
S_{j}^{A}(n)=\frac{\left(\frac{n}{2}-j\right) S_{j-1}^{A}(n)-\sum_{i=0}^{j-1} S_{i}^{A}(n)}{\frac{n}{2}+j}
$$

where $S_{0}^{A}=1$.

Observe that these coefficients are a decreasing function of $j$. Therefore they are all less than 1, which implies that $S_{j}^{A}(n) \sigma_{\frac{n}{2}}^{A}$ decreases in $j$. Furthermore at some point, $S_{i}^{A}$ becomes negative. Let $j_{A}^{*}$ denote the last positive coefficient. This value defines the support of candidate $A$ 's strategy in equilibrium in the following way. Since larger values of $j>j_{A}^{*}$, would imply $\sigma_{\frac{n}{2}-j}^{A}=S_{j}^{A}(n) \sigma_{\frac{n}{2}}^{A} \leq 0$. If $S_{j}^{A}(n)<0$, then this is is strictly negative and is not a feasible strategy, so we must set $\sigma_{\frac{n}{2}-j}^{A}=0$. for all $j>j_{A}^{*}$. If $S_{j_{A}^{*}+1}^{A}(n)=0$, then $\sigma_{\frac{n}{2}-j_{A}^{*}-1}^{A}$ is uniquely defined by $\sigma_{\frac{n}{2}-j_{A}^{*}-1}^{A}=S_{j_{A}^{*}+1}^{A}(n) \sigma_{\frac{n}{2}}^{A}=0$ and for all $j>j_{A+1}^{*}$, we have $S_{j}^{A}(n)<0$, again implying $\sigma_{\frac{n}{2}-j}^{A}=0$.

Thus, if $S_{j_{A}^{*}}^{A}(n)>0$ and $S_{j_{A}^{*}+1}^{A}(n) \leq 0$, then candidate $A$ 's support is the set of policies:

$$
\overline{\bar{\Sigma}}_{n}=\left\{x_{\frac{n}{2}-j^{*}}, x_{\frac{n}{2}-j^{*}+1}, \ldots, x_{\frac{n}{2}}, x_{\frac{n}{2}+1}, \ldots, x_{\frac{n}{2}+j^{*}}, x_{\frac{n}{2}+j^{*}+1}\right\} .
$$

Moreover, $\sigma_{\frac{n}{2}-j}^{A}$ is uniquely defined by $\sigma_{\frac{n}{2}-j}^{A}=S_{j}^{A}(n) \sigma_{\frac{n}{2}}^{A}$ for all $j=0, \ldots j^{*}$ and 
$\sigma_{\frac{n}{2}-j}^{A}=0$. for all $j>j_{A}^{*}$. When we substitute $\sigma_{\frac{n}{2}-j}^{A}=S_{j}^{A}(n) \sigma_{\frac{n}{2}}^{A}$ for all $j$ such that $S_{j}^{A}(n)$ is positive in the constraint that the sum of all mixing probabilities equals one $\left(\sum_{j=0}^{j^{*}} \sigma_{\frac{n}{2}-j}^{A}=\frac{1}{2}\right)$, we obtain a unique value for $\sigma_{\frac{n}{2}}^{A}$ :

$$
\sigma_{\frac{n}{2}}^{A}=\frac{1}{2\left(\sum_{0 \leq i \leq j^{*}} S_{i}^{A}(n)\right)}
$$

Substituting this into the equations $\sigma_{\frac{n}{2}-j}^{A}=S_{j}^{A}(n) \sigma_{\frac{n}{2}}^{A}$ for all $1 \leq j \leq j^{*}$ gives the unique symmetric probability distribution that solves the constraints of candidate $D$, thus it defines the mixed strategy of candidate $A$. Hence player $A$ has a unique symmetric no-gap equilibrium strategy, which proves the first part in the theorem.

For player $D$, we consider two cases:

Case $1\left(S_{j^{*}+1}^{A}(n)<0\right)$ :

In this case, by construction, player $A$ is mixing so that player $D$ is indifferent between all strategies in the support, $\overline{\bar{\Sigma}}_{n}$, and receives a strictly higher payoff from a strategy in $\overline{\bar{\Sigma}}_{n}$ than from any strategy not in $\overline{\bar{\Sigma}}_{n}$. Therefore, the support of $\sigma^{D}$ is either $\overline{\bar{\Sigma}}_{n}$ or a strict subset of $\overline{\bar{\Sigma}}_{n}$. Since $\sigma^{D}$ has no gaps, then in the latter case, it must be that $\sigma_{\frac{n}{2}-j^{*}}^{D}=0$. If so, player $A$ would be better off setting $\sigma_{\frac{n}{2}-j^{*}}^{A}=0$, a contradiction. Thus the support of $\sigma^{D}$ is $\overline{\bar{\Sigma}}_{n}$.

We can then use equation (1) to write $\sigma_{k}^{D}$ as a function of $\sigma_{i}^{D}$ with $k<i \leq \frac{n}{2}$, that is

$$
\sigma_{k}^{D}=\frac{(n-k) \sigma_{k+1}^{D}+\sum_{i=2}^{\frac{n}{2}} \sigma_{k+i}^{D}}{(k-1)}
$$

As before for all $1 \leq k<\frac{n}{2}$ we can write each $\sigma_{\frac{n}{2}-j}^{D}$ as a linear function of $\sigma_{\frac{n}{2}}^{D}$, that is, $\sigma_{\frac{n}{2}-j}^{D}=S_{j}^{D}(n) \sigma_{\frac{n}{2}}^{D}$ with coefficients:

$$
S_{j}^{D}(n)=\frac{\left(\frac{n}{2}+j\right) S_{j-1}^{D}(n)+\sum_{i=0}^{j-2} S_{i}^{D}(n)}{\frac{n}{2}-j-1}
$$

where $S_{0}^{D}=1$. 
Observe that these coefficients are an increasing function of $j$, therefore they are all larger than 1, which implies that the probability that each policy is assigned by candidate $D$ 's strategy increases as we move away from the center. From before we know that location $\frac{n}{2}-j^{*}$ is the left-most strategy assigned positive probability in $\bar{\sigma}^{D}$ Given this, we can construct $\bar{\sigma}^{D}$.

Given this, we can construct $\bar{\sigma}^{D}$ directly, by substituting $\sigma_{\frac{n}{2}-j}^{D}=S_{j}^{D}(n) \sigma_{\frac{n}{2}}^{D}$ for all $j \leq j^{*}$ and imposing the constraint that probabilities have to add up to one $\left(\sum_{j=0}^{j^{*}} \sigma_{\frac{n}{2}-j}^{D}=\frac{1}{2}\right)$. This gives a unique value for $\bar{\sigma}_{\frac{n}{2}}^{D}=\frac{1}{2\left(\sum_{0 \leq i \leq j^{*}} S_{i}^{D}(n)\right)}$. Substituting this value into the equations $\bar{\sigma}_{\frac{n}{2}-j}^{D}=S_{j}^{D}(n) \sigma_{\frac{n}{2}}^{D}$ for all $1 \leq j \leq j^{*}$ gives us the unique symmetric probability distribution that solves the constraints of candidate $A$, thus it defines the equilibrium mixed strategy of candidate $D$.

Case $2\left(S_{j^{*}+1}^{A}(n)=0\right)$ :

In this case, by construction, player $A$ is mixing so that player $D$ is indifferent not only between all strategies in the support, $\overline{\bar{\Sigma}}_{n}$, but also the next location just outside of $\overline{\bar{\Sigma}}_{n}$ (locations $\frac{n}{2}-j^{*}-1$ and $\frac{n}{2}+j^{*}+2$ ), which we denote $\overline{\bar{\Sigma}}_{n}^{+}$. Therefore, in this case, the support of $\sigma^{D}$ can be either $\overline{\bar{\Sigma}}_{n}$ or $\overline{\bar{\Sigma}}_{n}^{+}$. As in case $1, \bar{\sigma}^{D}$ is the unique solution on the support of $\overline{\bar{\Sigma}}_{n}$.

We can also construct, in a similar way, an equilibrium strategy for $D$ on the support $\overline{\bar{\Sigma}}_{n}^{+}$. Following a similar argument, one such strategy, $\bar{\sigma}^{D+}$ is found by setting $\bar{\sigma}_{\frac{n}{2}-j}^{D+}=S_{j}^{D}(n) \bar{\sigma}_{\frac{n}{2}}^{D+}$ for all $j \leq j^{*}+1, \bar{\sigma}_{\frac{n}{2}}^{D+}=\frac{1}{2\left(\sum_{0 \leq i \leq j^{*}+1} S_{i}^{D}(n)\right)}$. Since $S_{j}^{D}(n)>0$, for all $j$, this gives us a new symmetric nogap probability distribution for $D$, in which candidate $A$ is indifferent between all locations in $\bar{\Sigma}_{n}$. Next, observe that, since this is a constant sum game, the set of Nash equilibria is convex, so there are in fact a continuum of equilibria consisting of all convex combinations of $\left(\bar{\sigma}^{A}, \bar{\sigma}^{D}\right)$ and $\left(\bar{\sigma}^{A}, \bar{\sigma}^{D+}\right)$. For all of these equilibria, with the exception of $\left(\bar{\sigma}^{A}, \bar{\sigma}^{D+}\right)$, candidate $A$ is strictly better off choosing a location in $\overline{\bar{\Sigma}}_{n}$ than either of the locations $\frac{n}{2}-j^{*}-1$ and $\frac{n}{2}+j^{*}+2$.

This result does not rule out other equilibria which are either asymmetric or have gaps. We conjecture that there are no equilibria with gaps. 


\subsection{Examples}

Since the derivation above only applies to the case of $n>8$ we computed directly, for smaller odd values of $n$, the symmetric no-gap solutions where $\sigma^{A}$ and $\sigma^{D}$ have the same support. These are given in the table below. ${ }^{13}$ We also show a second symmetric equilibrium with no gaps for the case of $n=4$, to illustrate that the support of candidate $A$ 's mixed strategy can be strictly contained in the support of candidate $D$ 's mixed strategy. In fact, for $n=4$ there exists a continuum of equilibria, with $\sigma^{A}=(0, .5, .5,0)$ and $\sigma^{D}=(a, b, b, c)$, where $a \leq \frac{1+b}{2}$ and $c \leq \frac{1+b}{2}$, almost all of which are asymmetric.

\begin{tabular}{|c|c|c|}
\hline$n$ & $\sigma^{A}$ & $\sigma^{D}$ \\
\hline 4 & $0, .5, .5,0$ & $0, .5, .5,0$ \\
\hline 4 & $0, .5, .5,0$ & $.2, .3, .3, .2$ \\
\hline 6 & $0, .1, .4, .4, .1,0$ & $0, .4, .1, .1, .4,0$ \\
\hline 8 & $0,0, .14, .36, .36, .14,0,0$ & $0,0, .36, .14, .14, .36,0,0$ \\
\hline
\end{tabular}

\section{Properties of equilibrium for $n \rightarrow \infty$}

\subsection{Limiting properties of expected payoffs}

A natural question to ask is "How big an advantage does $A$ enjoy over $B$, in equilibrium?" In any mixed strategy equilibrium, all pure strategies in the support yield the same expected payoff. Therefore, to answer this question, we evaluate the equilibrium probabilities of winning for each of the two candidates, when they locate at $\frac{n}{2}$. Recall that for all $n>1$, both candidates mix with positive probability at $\frac{n}{2}$. That is, $\sigma_{\frac{n}{2}}^{D}>0$ and $\sigma_{\frac{n}{2}}^{A}>0$. Also, since this is a constant sum game, if there are any other equilibria (including asymmetric equilibria and/or equilibria with gaps), all such equilibria will yield the same expected payoff.

From equation (1), with $n$ locations the equilibrium expected payoffs for $A$ if strategy $\frac{n}{2}$ is used, denoted $\Pi_{n}^{A}$ are:

$$
\Pi_{n}^{A}=\frac{1}{2}+\frac{\sigma_{\frac{n}{2}}^{D}}{2}+\frac{1}{n} \sum_{i=1}^{k^{*}}(i+1) \sigma_{\frac{n}{2}-i}^{D} .
$$

Similarly, from equation (2), with $n$ locations the equilibrium expected payoffs for $D$ if strategy $\frac{n}{2}$ is used are:

\footnotetext{
${ }^{13}$ For $n$ odd, see the derivations in the appendix.
} 


$$
\Pi_{n}^{D}=\frac{1}{2}-\frac{\sigma_{\frac{n}{2}}^{A}}{2}+\frac{1}{n} \sum_{i=1}^{k^{*}} i \sigma_{\frac{n}{2}-i}^{A}
$$

This immediate delivers the following theorem, which states that the candidate with an a priori advantage is the more likely candidate to win.

Theorem 2: $\Pi_{n}^{A}>\frac{1}{2}>\Pi_{n}^{D}$ for all even $n=1,2, \ldots$

Proof: Since $\sigma_{\frac{n}{2}}^{D}>0$ and $\sigma_{i}^{D} \geq 0$, this implies that $\Pi^{A}=\frac{1}{2}+\frac{\sigma_{\frac{n}{2}}^{D}}{2}+\frac{1}{n} \sum_{i=1}^{k^{*}}(i+1) \sigma_{\frac{n}{2}-i}^{D}>$ $\frac{1}{2}$. Since $\Pi^{A}+\Pi^{D}=1$, this implies that $\Pi^{D}<\frac{1}{2}$.

Referring to the examples in Table 1, one can directly verify these formulas for small values of $n$. For example, $\Pi_{4}^{A}=\frac{3}{4}$ and $\Pi_{6}^{A}=\frac{41}{60}$. However, notice that $\Pi_{A}^{n}$ is not monotonically decreasing in $n$. Thus it is not clear how the size of the advantage to $A$ changes as $n$ changes. Thus, we next address the question: What is $\lim _{n \rightarrow \infty}\left\{\Pi_{n}^{A}\right\}$ ? This is an interesting question, since if we let $n$ get very large, we approach the standard Downsian model in which candidates locate on the $[0,1]$ continuum. Furthermore, as we take this limit the advantage to $A$ is infinitesimal, since we are assuming $\delta<\frac{1}{n-1}$. The following theorem proves that the advantage disappears in the limit. One can interpret this as an upper hemicontinuity result of equilibrium payoffs, as we let the magnitude of $A$ 's advantage $(\delta)$ go to 0 .

Theorem 3: $\lim _{n \rightarrow \infty}\left\{\Pi_{n}^{A}\right\}=\frac{1}{2}$.

Proof: In the proof we only consider limits of sequences of even values of $n$. A similar proof applies for the odd case. The expression on the RHS of (3) has three terms. What we prove is that the second and the third terms each converges to 0 in $n$, which leaves only the first term, implying that $\lim _{n \rightarrow \infty}\left\{\Pi_{n}^{D}\right\}=\frac{1}{2}$ and hence $\lim _{n \rightarrow \infty}\left\{\Pi_{n}^{A}\right\}=\frac{1}{2}$. To show that the second term converges to 0 requires proving that $\lim _{n \rightarrow \infty}\left\{\sigma_{\frac{n}{2}}^{A}\right\}=0$. Recall that, by construction, $\sigma_{\frac{n}{2}}^{A}=\frac{1}{2\left(\sum_{0 \leq i \leq j_{A}^{*}+1} S_{i}^{A}(n)\right)}$. Therefore, we need to show that $\sum_{0 \leq i \leq j_{A}^{*}+1} S_{i}^{A}(n)$ increases without bound as $n$ gets large. We do this by proving inductively that $\lim _{n \rightarrow \infty} S_{i}^{A}(n)=1$ for all $i$. First, observe that $S_{0}^{A}(n)=1$ for all $n$, and $S_{1}^{A}(n)=\frac{\frac{n}{2}+1}{\frac{n}{2}-2} \rightarrow 1$. Under the induction hypothesis, let $i$ be any integer greater than 1 and suppose that $S_{k}^{A}(n) \rightarrow 1$ for all $k<i$. We just need to show that 
this implies $S_{i}^{A}(n) \rightarrow 1$. By equation

$$
S_{i}^{A}(n)=\frac{\left(\frac{n}{2}-i\right) S_{i-1}^{A}(n)-\sum_{k=0}^{i-1} S_{k}^{A}(n)}{\frac{n}{2}+i}
$$

The right hand side converges to $S_{i-1}^{A}(n)$, which by hypothesis converges to 1 . Hence $\lim _{n \rightarrow \infty} S_{i}^{A}(n)=1$. Therefore, we have established that $\lim _{n \rightarrow \infty}\left\{\sigma_{\frac{n}{2}}^{A}\right\}=0$. Finally, we have to show that the third term of equation (3), $\frac{1}{n} \sum_{i=1}^{k^{*}} i \sigma_{\frac{n}{2}-i}^{A}$, converges to 0. Summing equations (3) and (4), gives us

$$
\begin{aligned}
\Pi_{n}^{A}+\Pi_{n}^{D} & =\left[\frac{1}{2}+\frac{\sigma_{\frac{n}{2}}^{D}}{2}+\frac{1}{n} \sum_{i=1}^{k^{*}}(i+1) \sigma_{\frac{n}{2}-i}^{D}\right]+\left[\frac{1}{2}-\frac{\sigma_{\frac{n}{2}}^{A}}{2}+\frac{1}{n} \sum_{i=1}^{k^{*}} i \sigma_{\frac{n}{2}-i}^{A}\right] \\
& =1+\frac{\sigma_{\frac{n}{2}-}^{D} \sigma_{\frac{n}{2}}^{A}}{2}+\frac{1}{n} \sum_{i=1}^{k^{*}}\left\{(i+1) \sigma_{\frac{n}{2}-i}^{D}+i \sigma_{\frac{n}{2}-i}^{A}\right\}
\end{aligned}
$$

¿From above, $\sigma_{\frac{n}{2}}^{A} \rightarrow 0$. The same inductive argument ${ }^{14}$ can be used to show $\sigma_{\frac{n}{2}}^{D} \rightarrow 0$, so the second term, $\frac{\sigma_{\frac{n}{2}}^{D}-\sigma_{\frac{n}{2}}^{A}}{2}$ vanishes, leaving:

$$
\Pi_{n}^{A}+\Pi_{n}^{D} \rightarrow 1+\frac{1}{n} \sum_{i=1}^{k^{*}}\left\{(i+1) \sigma_{\frac{n}{2}-i}^{D}+i \sigma_{\frac{n}{2}-i}^{A}\right\}
$$

However, by definition, $\Pi_{n}^{A}+\Pi_{n}^{D}=1$ for all $n$. Therefore, $\frac{1}{n} \sum_{i=1}^{k^{*}}\left\{(i+1) \sigma_{\frac{n}{2}-i}^{D}+\right.$ $\left.i \sigma_{\frac{n}{2}-i}^{A}\right\} \rightarrow 0$. Since $\sigma_{\frac{n}{2}-i}^{D}$ and $\sigma_{\frac{n}{2}-i}^{A}$ are both nonnegative for all $i$, this implies that both $\frac{1}{n} \sum_{i=1}^{k^{*}} i \sigma_{\frac{n}{2}-i}^{A}$ and $\sum_{i=1}^{k^{*}}(i+1) \sigma_{\frac{n}{2}-i}^{D}$ converge to 0 .

This establishes that $\lim _{n \rightarrow \infty}\left\{\Pi_{n}^{D}\right\}=\frac{1}{2}$ and therefore $\lim _{n \rightarrow \infty}\left\{\Pi_{n}^{A}\right\}=\frac{1}{2}$.

\footnotetext{
${ }^{14}$ This also follows directly, since we already showed earlier that $0<\sigma_{\frac{n}{2}}^{D}<\sigma_{\frac{n}{2}}^{A}$.
} 


\subsection{Limiting properties of the mixed strategy support}

We can show that the proportion of locations that are used with positive probability in equilibrium goes to zero in $n$.

Theorem 4: $\lim _{n \rightarrow \infty} k^{*}(n) / n=\frac{1}{2}$.

Proof:To simplify the proof we go directly to the limit where the policy space is the $[0,1]$ interval, and the $n / 2$ location corresponds to $1 / 2$ and $\delta$ is arbitrarily small. ${ }^{15}$ By definition, $\lim _{n \rightarrow \infty} k^{*}(n) / n \leq \frac{1}{2}$. Suppose that $\liminf _{n \rightarrow \infty} k^{*}(n) / n<\frac{1}{2}$. Then, in the limiting case we are considering, this means that there is a subsequence such that the (common) support of the two candidates' equilibrium strategies along this subsequence converges to $1 / 2-\varepsilon$. We now show that this cannot be an equilibrium. Consider the strategy for candidate $A$ that places all weight on $1 / 2$ while candidate $D$ uses some u-shaped strategy $\sigma_{\infty}^{D}$ with support on $[1 / 2-\varepsilon, 1 / 2+\varepsilon]$. Then, by symmetry, the expected payoff to $A$ is

$$
\begin{aligned}
\Pi_{\infty}^{A} & =2 \int_{1 / 2}^{1 / 2+\varepsilon} \frac{1 / 2+t}{2} \sigma_{\infty}^{D}(t) d t \\
& =1 / 4+\int_{1 / 2}^{1 / 2+\varepsilon} t \sigma_{\infty}^{D}(t) d t \\
& \geq 1 / 4+1 / 4+\varepsilon / 4 \\
& >1 / 2
\end{aligned}
$$

which contradicts the result in the previous section.

This, together with the previous result about expected payoffs implies that the solution converges to the standard median voter result. Candidates' policies converge to the median and each candidate expects to win half the time.

\section{Extensions}

In this section, we look at three extensions of the model. The results of the previous sections were derived assuming that the $A$ 's advantage was small. That is, voters closer to $D$ than to $A$ always vote for $D$, so the quality advantage enjoyed by $A$ only came into play when a voter was equidistant from $A$ and $D$. The first extension

\footnotetext{
${ }^{15}$ That is, voters will vote for the closest candidate unless they are equidistant, in which case they will vote for $A$.
} 
relaxes the assumption that the distribution of median voter's ideal point is uniform. The second extension we consider retains the assumption of a finite policy space, but considers what happens if $\delta$ is slightly larger, so that some voters will vote for $A$ over $D$, even if $D$ is closer. The third extension relaxes the assumption of a finite policy space, also considering the case of larger $\delta$, but where the policy space is the $[0,1]$ interval.

\subsection{Non-uniform distribution of voters}

\subsubsection{Voters in three positions}

Let $n=3$, so there are 3 possible locations, $a, b$ or $c$, where $a, b, c \in R$ and $a<b<c$. The probability the median voter is located at ideal point in $a$ is denoted by $\alpha$, similarly the probabilities she is located at ideal points $b$ or $c$ are denoted by $\beta$ and $\gamma$ respectively, with $\alpha+\beta+\gamma=1$. Suppose that the utility functions of the voters are as the described in the previous sections, and assume that $0<\delta<\max \{a-b, b-c\}$

The payoffs for the game played by the parties appear in the table below:

\begin{tabular}{c|c|c|c|}
\multicolumn{1}{c}{$a$} & \multicolumn{1}{c}{$b$} & $c$ \\
\cline { 2 - 4 }$a$ & 1,0 & $\alpha, 1-\alpha$ & $\alpha+\beta, 1-\alpha-\beta$ \\
\cline { 2 - 4 }$b$ & $1-\alpha, \alpha$ & 1,0 & $\alpha+\beta, 1-\alpha-\beta$ \\
\cline { 2 - 4 }$c$ & $1-\alpha, \alpha$ & $1-\alpha-\beta, \alpha+\beta$ & 1,0 \\
\cline { 2 - 4 } & &
\end{tabular}

where $A$ is the row player and $D$ is the column player. For any mixed strategy by $D$, denoted $\sigma^{D}$, $A$ 's expected payoffs for the three possible pure strategies are:

$$
\begin{aligned}
& \pi^{A}\left(a, \sigma^{D}\right)=\sigma_{a}^{D}+\alpha \sigma_{b}^{D}+(\alpha+\beta) \sigma_{c}^{D} \\
& \pi^{A}\left(b, \sigma^{D}\right)=(1-\alpha) \sigma_{a}^{D}+\sigma_{b}^{D}+(\alpha+\beta) \sigma_{c}^{D} \\
& \pi^{A}\left(c, \sigma^{D}\right)=(1-\alpha) \sigma_{a}^{D}+(1-\alpha-\beta) \sigma_{b}^{D}+\sigma_{c}^{D}
\end{aligned}
$$

To solve for a totally mixed strategy equilibrium we equate these expected payoffs. For example, equating the expected payoffs for $A$, we get

$$
\begin{aligned}
& \pi^{A}\left(a, \sigma^{D}\right)=\pi^{A}\left(b, \sigma^{D}\right) \Longrightarrow \alpha \sigma_{a}^{D}+(\alpha-1) \sigma_{b}^{D}=0 \\
& \pi^{A}\left(b, \sigma^{D}\right)=\pi^{A}\left(c, \sigma^{D}\right) \Longrightarrow(\alpha+\beta) \sigma_{b}^{D}+(\alpha+\beta-1) \sigma_{c}^{D}=0
\end{aligned}
$$

Thus the equilibrium value of $\sigma^{D}$ can be determined by the following system of 3 
equations:

$$
\begin{aligned}
\alpha \sigma_{a}^{D}+(\alpha-1) \sigma_{b}^{D} & =0 \\
(\alpha+\beta) \sigma_{b}^{D}+(\alpha+\beta-1) \sigma_{c}^{D} & =0 \\
\sigma_{a}^{D}+\sigma_{b}^{D}+\sigma_{c}^{D} & =1
\end{aligned}
$$

The solution is:

$$
\sigma_{a}^{D}=\frac{(1-\alpha)-(1-\alpha)(\alpha+\beta)}{1-(1-\alpha)(\alpha+\beta)}, \sigma_{b}^{D}=\frac{\alpha-\alpha(\alpha+\beta)}{1-(1-\alpha)(\alpha+\beta)}, \sigma_{c}^{D}=\frac{\alpha(\alpha+\beta)}{1-(1-\alpha)(\alpha+\beta)}
$$

Solving for $\sigma^{A}$ in a similar way gives:

$$
\sigma_{a}^{A}=\frac{(\alpha+\beta)^{2}-\beta}{1-(1-\alpha)(\alpha+\beta)}, \sigma_{b}^{A}=\frac{(1-\alpha)(\alpha+\beta)-\beta^{2}}{1-(1-\alpha)(\alpha+\beta)}, \sigma_{c}^{A}=\frac{(1-\alpha)^{2}-\beta}{1-(1-\alpha)(\alpha+\beta)} .
$$

The solution to $\sigma^{A}$ is non-negative if and only if $(1-\alpha)^{2} \geq \beta$ and $(\alpha+\beta)^{2} \geq \beta$. If the first of these inequalities is violated, then the equilibrium involves mixing only over strategies $a$ and $b$, and if the second of these inequalities is violated, then the equilibrium involves mixing only over strategies $b$ and $c$. The symmetric case, $\alpha=\gamma$, is natural special case to consider. With symmetry, $\beta=1-2 \alpha$, so we are guaranteed that $(1-\alpha)^{2} \geq \beta$ and $(\alpha+\beta)^{2} \geq \beta$. The solution can be written as:

$$
\begin{aligned}
\sigma_{a}^{A} & =\sigma_{c}^{A}=\frac{\alpha}{2-\alpha}, \sigma_{b}^{A}=\frac{2-3 \alpha}{2-\alpha} \\
\sigma_{a}^{D} & =\sigma_{c}^{D}=\frac{1-\alpha}{2-\alpha}, \sigma_{b}^{D}=\frac{\alpha}{2-\alpha}
\end{aligned}
$$

This equilibrium solution has several interesting properties. First note that since $\alpha \leqslant \frac{1}{2}$ this implies that $\sigma_{a}^{A} \leqslant \sigma_{b}^{A}$ and $\sigma_{a}^{D} \geqslant \sigma_{b}^{D}$. In other words, the advantaged candidate places more weight in the central location than does the disadvantaged candidate. Furthermore, the comparative statics are interesting. As one would expect, $\frac{\partial \sigma_{a}^{A}}{\partial \alpha}>0$ so that as the electorate becomes more dispersed the advantaged candidate moves away from the central location. Less intuitive is the result that $\frac{\partial \sigma_{a}^{D}}{\partial \alpha}<0$, implying that the disadvantaged candidate moves toward the center as it becomes less likely the median is located in the center. At the extreme case, when $\alpha=\frac{1}{2}$ (i.e. zero probability that the median is in the center), both candidates mix uniformly over the three locations. In this case, $D$ wins $1 / 3$ of the time. At the opposite extreme, 
as $\alpha$ approaches $0, A$ places all probability at $1 / 2$ and $D$ places all probability at the extremes. The probability that $D$ wins, converges to 0 .

\subsubsection{Voters in four positions}

Consider the previous model with four positions, $a<b<c<r$, with median voter probabilities given by $\alpha, \beta, \gamma$, and $\rho$, respectively. And consider the symmetric case in which $\alpha=\rho, \beta=\gamma$, and $\alpha+\beta=\frac{1}{2}$. The reduced form game played by the parties in this case is:

\begin{tabular}{|c|c|c|c|c|}
\hline & $a$ & $b$ & $c$ & $r$ \\
\hline$a$ & 1,0 & $\alpha, 1-\alpha$ & $\frac{1}{2}, \frac{1}{2}$ & $\frac{1}{2}, \frac{1}{2}$ \\
\hline b & $1-\alpha, \alpha$ & 1,0 & $\frac{1}{2}, \frac{1}{2}$ & $1-\alpha, \alpha$ \\
\hline$c$ & $1-\alpha, \alpha$ & $\frac{1}{2}, \frac{1}{2}$ & 1,0 & $1-\alpha, \alpha$ \\
\hline & $\frac{1}{2}, \frac{1}{2}$ & $\frac{1}{2}, \frac{1}{2}$ & $\alpha, 1-\alpha$ & 1,0 \\
\hline
\end{tabular}

We obtain the following equilibrium mixing probabilities as a function of $\alpha:^{16}$

$$
\begin{aligned}
& \sigma_{a}^{A}=\max \left\{\frac{2 \alpha-\frac{1}{2}}{2 \alpha+1}, 0\right\} \\
& \sigma_{b}^{A}=\min \left\{\frac{1-\alpha}{2 \alpha+1}, \frac{1}{2}\right\} \\
& \sigma_{a}^{D}=\min \left\{\frac{1-\alpha}{2 \alpha+1}, \frac{1}{2}\right\} \\
& \sigma_{b}^{D}=\max \left\{\frac{2 \alpha-\frac{1}{2}}{2 \alpha+1}, 0\right\}
\end{aligned}
$$

Thus, the results are similar to the 3 location case. As before, since $\alpha \leqslant \frac{1}{2}$, we have $\sigma_{a}^{A} \leqslant \sigma_{b}^{A}$ and $\sigma_{a}^{D} \geqslant \sigma_{b}^{D}$ and $\frac{\partial \sigma_{a}^{A}}{\partial \alpha}>0, \frac{\partial \sigma_{a}^{D}}{\partial \alpha}<0$. Notice that if $\alpha<\frac{1}{4}$, we have $\sigma_{a}^{A}=0, \sigma_{b}^{A}=\frac{1}{2}, q \sigma_{a}^{D}=\frac{1}{2}, \sigma_{b}^{D}=0$, so the advantaged candidate places all weight in the two central locations, while the disadvantaged candidate places all weight at the extremes.

\footnotetext{
${ }^{16}$ When $\alpha=.25$ there are multiple equilibria, which are described in section 3.4. When $\alpha<25$ there are also multiple equilibria, all of which share the property that $D$ puts all weight on the outside and $A$ mixes $(.5, .5)$ over the two central locations.
} 


\subsection{Larger $\delta: 1<(n-1) \delta<2$}

Next we consider the case candidate $A$ wins not only when the median voter is equidistant or closer to $A$ than to $D$, but also $A$ wins if $D$ is closer to the median voter by only $1 /(n-1)$. That is, $1<(n-1) \delta<2$. It turns out that in this case there are gaps in the mixed strategy distribution, asymmetries, and non-monotonicities, which makes it difficult to derive a solution using the same algorithm as above.

The payoffs of candidate $A$ for a given strategy $i$ can be written as:

$$
\begin{aligned}
\pi_{A}\left(x_{i}, \sigma^{D}\right)= & \ldots+(n-i+3) \sigma_{i-4}^{D}+(n-i+3) \sigma_{i-3}^{D}+(n-i+2) \sigma_{i-2}^{D}+n \sigma_{i-1}^{D} \\
& +n \sigma_{i}^{D}+n \sigma_{i+1}^{D}+(i+1) \sigma_{i+2}^{D}+(i+2) \sigma_{i+3}^{D}+(i+2) \sigma_{i+4}^{D}+\ldots
\end{aligned}
$$

The payoffs of candidate $D$ for a given strategy $i$ can be written as:

$$
\begin{aligned}
\pi_{D}\left(x_{i}, \sigma^{A}\right)= & \ldots+(n-i+2) \sigma_{i-4}^{A}+(n-i+1) \sigma_{i-3}^{A}+(n-i+1) \sigma_{i-2}^{A}+(i) \sigma_{i+2}^{A} \\
& +(i) \sigma_{i+3}^{A}+(i+1) \sigma_{i+4}^{A}+(i+1) \sigma_{i+5}^{A}+\ldots
\end{aligned}
$$

To check if we can use our recursive method of equating payoffs from adjacent strategies, we used the Gambit (1999) game solver software to compute the equilibrium of the game, for a range of low values of $n$. The results are reported in the table below, which demonstrates that there are gaps in the equilibrium strategy of $D$. We do not know whether there can be gaps in the equilibrium strategy of $A$. There are other peculiarities that are troubling, and suggest that our methods for the case of small $\delta$ will be difficult to apply. For example, the equilibrium is not necessarily symmetric, in which case there exist equilibria in pairs, which are mirror images of each other. Also, to obtain limiting result that are different from the results for small $\delta$, we would have to consider values of $\delta$ that increase as $n$ increases. Otherwise, for example if we require $1<(n-1) \delta<2$ for all $n, \delta$ will be driven to 0 in the limit, so we will effectively be back in the small $\delta$ case. Thus, we also computed some examples for higher ranges of $\delta$ and find that the gaps proliferate as $\delta$ increases. Because of these kinds of problems, we are unable to obtain a general solution for the large $\delta$ case with a large finite number of locations. 


\begin{tabular}{|c|c|c|}
\hline $\mathrm{n}$ & $\sigma^{A}$ & $\sigma^{D}$ \\
\hline 3 & $0,1,0$ & $1,0,0$ \\
\hline 4 & $1 / 4,1 / 4,1 / 2,0$ & $1 / 2,0,0,1 / 2$ \\
\hline 5 & $0,1 / 3,1 / 3,1 / 3,0$ & $2 / 3,0,0,1 / 3,0$ \\
\hline 6 & $0,1 / 3,1 / 6,1 / 6,1 / 3,0$ & $0,1 / 2,0,0,1 / 2,0$ \\
\hline 7 & $0,1 / 7,2 / 7,1 / 7,2 / 7,1 / 7,0$ & $3 / 7,0,1 / 7,0,0,3 / 7,0$ \\
\hline 8 & $0,0,2 / 5,1 / 15,2 / 15,2 / 5,0,0$ & $0,4 / 15,0,1 / 5,0,0,8 / 15,0$ \\
\hline 10 & $0,0, .13, .18, .18, .18, .18, .13,0,0$ & $0, .31, .13,0, .05, .05,0, .13, .31,0$ \\
\hline 12 & $0,0,0, .19, .10, .20, .20, .10, .19,0,0,0$ & $0,0, .26, .15,0, .07, .07,0, .15, .26,0,0$ \\
\hline 24 & $\ldots, 0, .009, .08, .05, .07, .17, .10, .10, .17, .07, \ldots$ & $\ldots . .0, .20, .13, .01, .05, .04,0, .03, .03,0, .04, \ldots$ \\
\hline
\end{tabular}

\subsection{Continuous locations}

The approach in the rest of this paper was to study a model with a finite number of feasible locations, and a small advantage. The findings above, for larger $\delta$, suggest that a different approach may be required to get further results. A natural alternative approach is the continuous location model, where the policy space, $\wp$, consists of all points in the $[0,1]$ interval. As before, suppose that the median voter is uncertain, and the prior beliefs of the candidates over the location of the median voter are uniform on $[0,1]$, and candidates maximize the probability of winning. The proof of Theorem 1 , that pure strategy equilibria fail to exist unless $\delta=0$, still applies.

While a mixed strategy equilibrium is guaranteed with finite locations, a mixed strategy equilibrium is not necessarily guaranteed to exist by the standard theorem for games with continuous payoffs (Glicksburg 1952), since the payoff function is discontinuous. The problem is related to the kind of discontinuity that arises in a game studied by Sion and Wolfe (1957), and later by Dasgupta and Maskin (1986). In this game, however, the discontinuities satisfy the technical condition of weak lower semi-continuity, as well as upper hemi-continuity of the sum of payoffs, and payoff discontinuities are restricted to a special (small) subset of strategy profiles. Therefore, we can appeal directly to Theorem 5 of Dasgupta and Maskin (1986, p.14) to establish existence of mixed strategy equilibrium in this game.

Theorem 5: Let $\wp=[0,1]$ and $\delta>0$. A Nash equilibrium point exists in mixed strategies.

Proof: See Appendix $B$.

The theorem can be proved under much less stringent assumptions. For example, the proof given in the appendix is easily adapted to allow for non-uniform distri- 
butions with continuous, strictly increasing CDF's. Preferences do not have to be "tent" preferences, and need not even be Euclidean, provided they are single peaked. One could also allow for heterogeneity of $\delta$ across voters, but at considerable cost of notation.

\section{Conclusions}

This paper has taken a first step toward solving for the mixed strategy equilibrium of a simple game between two candidates, in one dimension where one of the candidates has an advantage, and candidates only care about electoral success. ${ }^{17}$ Because of payoff discontinuities in the continuous location formulation of the problem, we look at equilibrium in a discrete policy space. We find that the advantaged candidate will locate more centrally than the disadvantaged candidate, and always has an equilibrium advantage, i.e. $A$ wins with probability greater than .5 .

However, if we look at the limiting case where the advantage becomes arbitrarily small (and the discrete grid on the policy space becomes arbitrarily fine), we obtain a continuity result: the distribution of the strategies of both $A$ and $D$ approaches a degenerate distribution with all mass at the median of the distribution, and each candidate wins with probability .5. Thus, even though pure strategy equilibrium disappears for any positive value of $\delta$, however small, this does not imply that the standard Downsian model is a knife edge case. It is a good approximation of equilibrium candidate locations as long as the advantage is small.

Examples suggest that some of these results may generalize to non-uniform distributions. For the case a coarse grid of strategies, we obtain some intuitive comparative statics results with symmetric non-uniform distributions. In particular, we find that as the distribution of the median voter becomes more certain, the equilibrium advantage of $A$ increases. And, as we know from Ansolabehere and Snyder (2000), in the limit $A$ wins with probability 1 , when there is no uncertainty. In the other direction, when the variance of the distribution of the median voter is maximal, $D$ has a fair chance $(1 / 3)$ of winning.

We also prove existence of equilibrium for the continuous location problem, for arbitrary values of $\delta$. A natural next step, is to solve for the mixed equilibrium in the continuous location setting.

\footnotetext{
${ }^{17}$ As explained before, one can view this either as a model either of vote maximizing candidates in a world where the locations of voters are common knowledge, or a model where candidates maximize the probability of winning with uncertainty about the location of the median voter.
} 
There are some interesting possibilities for embedding this model of candidate advantages into more complex and realistic models of campaigns. For example, Wittman (2000) has shown how one can include factors such as endorsements to signal quality to the voter. One can also look at the effects of campaign spending and advertising campaigns to affect voter beliefs about candidate quality. This would add a stage at the beginning of the game we studied here, in which candidates can choose spending levels, and the advantage of candidate $A$ will then depend on the spending levels of the two candidates. This would, in effect, endogenize $\delta$, which we assumed here to be exogenous. Such an extension would result in a combination of the spending game approach of Erikson and Palfrey (2000) with the asymmetric competition approach presented here. 


\section{REFERENCES}

Adams, James. 1999. Policy Divergence in Multicandidate Probabilistic Spatial Voting. Public Choice. 100: 103-22.

Ansolabehere, Stephen and James M. Snyder, Jr. 2000. Valence Politics and Equilibrium in Spatial Election Models. Public Choice. 103(June): 327-336.

Banks, Jeffrey S. and D. Roderick Kiewiet. 1989. Explaining Patterns of Candidate Competition in Congressional Elections. American Journal of Political Science 33: $997-1015$

Berger, Mark. M., Michael C. Munger, and Richard F. Potthoff. 2000. With Uncertainty, the Downsian Model Predicts Divergence. Journal of Theoretical Politics. 12 (April): 262-68.

Bernhardt, Daniel and Daniel Ingberman. 1985. Candidate Reputations and the Incumbency Effect. Journal of Public Economics. 27: 47-67.

Dasgupta, Partha and Eric Maskin. 1986. The Existence of Equilibrium in Discontinuous Economic Games I: Theory. Review of Economic Studies. 53: 1-26.

Downs, Anthony. 1957. An Economic Theory of Democracy. New York: Harper and Row.

Erikson, Robert and Thomas R. Palfrey. 2000. Equilibrium Effects in Campaign Spending Games: Theory and Data. American Political Science Review. (forthcoming, September issue)

GAMBIT software. 1999. http://www.hss.caltech.edu/gambit.

Glicksberg, I. L. 1952. A Further Generalization of the Kakutani Fixed Point Theorem with Application to Nash Equilibrium Points. Proceedings of the American Mathematical Society. 38: 170-74.

Groseclose, Tim. 1999. Character, Charisma, and Candidate Locations: Downsian Models when One Candidate Has a Valence Advantage. Draft. Stanford University.

Ingberman, Daniel. 1992. Incumbent Reputations and Ideological Campaign Contributions in Spatial Competition. Mathematical and Computer Modeling. 16: $147-69$. 
Kiewiet, D. Roderick. 1983. Macroeconomics and Micropolitics. Chicago: University of Chicago Press.

Kiewiet, D. Roderick and Langche Zeng. 1993. "An Analysis of Congressional Career Decisions, 1947-86". American Political Science Review 87: 928-41

Londregan, John and Thomas Romer. 1993. Polarization, Incumbency, and the Personal Vote. in Political Economy: Institutions, Competition, and Representation (W.A. Barnett, M.J. Hinich, and N.J. Schofield eds.). Cambridge: Cambridge University Press. 355-77.

Popkin, Samuel, John W. Gorman, Charles Phillips, and Jeffrey A. Smith. 1976. Comment: What Have You Done for Me Lately? Toward an Investment Theory of Voting. American Political Science Review. 70: 779-805.

Sion, M. and P. Wolfe. 1957. On a Game Without a Value. Contributions to the Theory of Games, III. (Princeton: Annals of Mathematical Studies No. 39), 299-306.

Stokes, Donald E. 1963. Spatial Models of Party Competition. American Political Science Review. 57: 368-77.

Wittman, Donald. 2000. Candidate Quality, Pressure Group Endorsements, and Uninformed Voters. draft. UC Santa Cruz. 


\section{Appendix A: $n$ odd}

We will show by construction the existence of symmetric equilibria with no gaps for the case of small $\delta$ when the policy space $\wp$ consists of an odd number of locations $n>7$ and $\rho$ is uniform. We also show that for large $n$ the equilibrium strategies approach the ones found for the case when $n$ is even.

As before, symmetric equilibria with no gaps are solved by first equating the payoffs of adjacent locations, and then finding one of the endpoints of the support.

If we denote by $k_{A}^{*}$ the first location in the support of $A^{\prime} s$ strategy, then we have a collection of $n-2 k_{A}^{*}+1$ equations of the form:

$$
\begin{aligned}
k \sigma_{k}^{D}+\sum_{i=1}^{\left[\frac{k-1}{2}\right]} \sigma_{k-2 i}^{D} & =(n-k) \sigma_{k+1}^{D}+\sum_{i=1}^{\left[\frac{n-k-1}{2}\right]} \sigma_{k+2 i+1}^{D} \\
\text { for } k_{A}^{*} & \leq k \leq n-k_{A}^{*}
\end{aligned}
$$

where the partial sums continue up to the point where the subscripts become either less than 1 or greater than $n$.

If we further assume symmetry, that is, $\sigma_{k}^{D}=\sigma_{n-k+1}^{D}$ we obtain the following system of equations that can be solved to obtain the mixing probabilities $\sigma^{D}$ :

$$
\left(\frac{n+1}{2}-j-1\right) \sigma_{\frac{n+1}{2}+j}^{D}=\left(\frac{n+1}{2}+j-1\right) \sigma_{\frac{n+1}{2}+j-1}^{D}+\sigma_{\frac{n+1}{2}}^{D}+\sum_{i=0}^{j-2} \sigma_{\frac{n+1}{2}+i}^{D}+(-1)^{j}\left(H_{o}^{D}-H_{e}^{D}\right)
$$

for $0 \leq j \leq \frac{n+1}{2}$, where

$$
\begin{aligned}
& H_{o}^{D}=\sum_{i=1}^{\left[\frac{n+1}{4}\right]} \sigma_{\frac{n+1}{2}+2 i-1}^{D} \\
& H_{e}^{D}=\sum_{i=1}^{\left[\frac{n-1}{4}\right]} \sigma_{\frac{n+1}{2}+2 i}^{D}
\end{aligned}
$$

Similarly, equating candidate $D^{\prime} s$ payoffs on the support of $D^{\prime} s$ strategy, we obtain a collection of $n-2 k_{D}^{*}+1$ equations of the form: 


$$
k \sigma_{k+1}^{A}+\sum_{i=1}^{\left[\frac{k}{2}\right]} \sigma_{k-2 i+1}^{A}=(n-k) \sigma_{k}^{A}+\sum_{i=1}^{\left[\frac{n-k}{2}\right]} \sigma_{k+2 i}^{A}
$$

for $k_{D}^{*} \leq k \leq n-k_{D}^{*}$, where the partial sums continue up to the point where the subscripts become either less than 1 or greater than $n$.

Imposing symmetry, $\sigma_{k}^{A}=\sigma_{n-k+1}^{A}$ we obtain the mixing probabilities of $A$ :

$$
\left(\frac{n+1}{2}+j-1\right) \sigma_{\frac{n+1}{2}+j}^{A}=\left(\frac{n+1}{2}-j-1\right) \sigma_{\frac{n+1}{2}+j-1}^{A}-\sum_{i=1}^{j-2} \sigma_{\frac{n+1}{2}+i}^{A}+(-1)^{j}\left(H_{o}^{A}-H_{e}^{A}\right)
$$

for $0 \leq j \leq j^{*} \leq \frac{n+1}{2}$ where

$$
\begin{aligned}
& H_{o}^{A}=\sum_{i=1}^{\left[\frac{n+1}{4}\right]} \sigma_{\frac{n+1}{2}+2 i-1}^{A} \\
& H_{e}^{A}=\sum_{i=1}^{\left[\frac{n-1}{4}\right]} \sigma_{\frac{n+1}{2}+2 i}^{A}
\end{aligned}
$$

These equations can also be solved recursively as in the even case to get the mixing probabilities of candidate $D$ :

$$
\begin{aligned}
\sigma_{\frac{n+1}{2}+1}^{D} & =\frac{\frac{n+1}{2} \sigma_{\frac{n+1}{2}}^{D}-\left(H_{o}^{D}-H_{e}^{D}\right)}{\frac{n+1}{2}-2} \\
\sigma_{\frac{n+1}{2}+2}^{D}= & \frac{\left(\frac{n+1}{2}+1\right) \sigma_{\frac{n+1}{2}+1}^{D}+\left(H_{o}^{D}-H_{e}^{D}\right)}{\frac{n+1}{2}-3} \\
\sigma_{\frac{n+1}{2}+3}^{D}= & \frac{\sigma_{\frac{n+1}{2}}^{D}+\sigma_{\frac{n+1}{2}+1}^{D}+\left(\frac{n+1}{2}+2\right) \sigma_{\frac{n+1}{2}+2}^{D}-\left(H_{o}^{D}-H_{e}^{D}\right)}{\frac{n+1}{2}-4} \\
& \ldots
\end{aligned}
$$

and so forth.

And the mixing probabilities of candidate $A$ : 


$$
\begin{aligned}
\sigma_{\frac{n+1}{2}+1}^{A}= & \frac{\left(\frac{n+1}{2}-1\right) \sigma_{\frac{n+1}{2}}^{A}-\left(H_{o}^{A}-H_{e}^{A}\right)}{\frac{n+1}{2}} \\
\sigma_{\frac{n+1}{2}+2}^{A}= & \frac{-\sigma_{\frac{n+1}{2}}^{A}+\left(\frac{n+1}{2}-3\right) \sigma_{\frac{n+1}{2}+1}^{A}+\left(H_{o}^{A}-H_{e}^{A}\right)}{\frac{n+1}{2}+1} \\
\sigma_{\frac{n+1}{2}+3}^{A}= & \frac{-\sigma_{\frac{n+1}{2}+1}^{A}+\left(\frac{n+1}{2}-4\right) \sigma_{\frac{n+1}{2}+2}^{A}-\left(H_{o}^{A}-H_{e}^{A}\right)}{\frac{n+1}{2}+2}
\end{aligned}
$$

and so forth.

Thus, we can write the probabilities that define the mixed strategy of candidate $A$ as

$$
\sigma_{\frac{n+1}{2}+j}^{A}=\phi_{j} \sigma_{\frac{n+1}{2}}^{A}+\beta_{j}\left(H_{o}^{A}-H_{e}^{A}\right)
$$

where $\phi_{0}=1, \phi_{1}=\frac{\frac{n+1}{2}-1}{\frac{n+1}{2}}$, and for $1<j<\frac{n+1}{2}$

$$
\begin{aligned}
\phi_{j} & =\frac{\left(\frac{n+1}{2}-j-1\right) \phi_{j-1}-\sum_{i=0}^{j-2} \phi_{i}}{\frac{n+1}{2}+j-1} \text { if } j>1 \text { is even } \\
& =\frac{\left(\frac{n+1}{2}-j-1\right) \phi_{j-1}-\sum_{i=1}^{j-2} \phi_{i}}{\frac{n+1}{2}+j-1} \text { if } j>1 \text { is odd }
\end{aligned}
$$

and $\beta_{0}=0, \beta_{1}=\frac{-1}{\frac{n+1}{2}}$, and for $1<j<\frac{n+1}{2}$

$$
\beta_{j}=\frac{\left(\frac{n+1}{2}-j-1\right) \beta_{j-1}+(-1)^{j}-\sum_{i=1}^{j-2} \beta_{i}}{\frac{n+1}{2}+j-1}
$$

And we can write the probabilities that define the mixed strategy of candidate $D$ as

$$
\sigma_{\frac{n+1}{2}+j}^{D}=\varphi_{j} \sigma_{\frac{n+1}{2}}^{D}+\psi_{j}\left(H_{o}^{D}-H_{e}^{D}\right)
$$


where $\varphi_{0}=1, \varphi_{1}=\frac{\frac{n+1}{2}}{\frac{n+1}{2}-2}$, and for $1<j<\frac{n+1}{2}$

$$
\begin{aligned}
\varphi_{j} & =\frac{\left(\frac{n+1}{2}+j-1\right) \varphi_{j-1}+\sum_{i=0}^{j-2} \varphi_{i}}{\frac{n+1}{2}-j-1} \text { if } j>1 \text { is odd } \\
& =\frac{\left(\frac{n+1}{2}+j-1\right) \varphi_{j-1}+\sum_{i=1}^{j-2} \varphi_{i}}{\frac{n+1}{2}-j-1} \text { if } j>1 \text { is even }
\end{aligned}
$$

And $\psi_{0}=0, \psi_{1}=-\frac{1}{\frac{n+1}{2}-2}$, and for $1<j<\frac{n+1}{2}$

$$
\psi_{j}=\frac{\left(\frac{n+1}{2}+j-1\right) \psi_{j-1}+(-1)^{j}+\sum_{i=1}^{j-2} \psi_{i}}{\frac{n+1}{2}-j-1}
$$

Since

$$
H_{o}^{A}-H_{e}^{A}=\sum_{i=1}^{\left[\frac{n+1}{4}\right]} \sigma_{\frac{n+1}{2}+2 i-1}^{A}-\sum_{i=1}^{\left[\frac{n-1}{4}\right]} \sigma_{\frac{n+1}{2}+2 i}^{A}
$$

and using the expressions above, we obtain that

$$
H_{o}^{A}-H_{e}^{A}=\frac{\sum_{i=1}^{\left[\frac{n}{2}\right]}(-1)^{i+1} \phi_{i}}{1-\sum_{i=1}^{\left[\frac{n}{2}\right]}(-1)^{i+1} \beta_{i}} \sigma_{\frac{n+1}{2}}^{A}
$$

Now we can substitute this into the previous expressions for the $\sigma^{A}$ 's and obtain that for $1 \leqslant j<\frac{n+1}{2}$

$$
\sigma_{\frac{n+1}{2}+j}^{A}=\left[\phi_{j}-\beta_{j} \frac{\sum_{i=1}^{\left[\frac{n}{2}\right]}(-1)^{i} \phi_{i}}{1+\sum_{i=1}^{\left[\frac{n}{2}\right]}(-1)^{i} \beta_{i}}\right] \sigma_{\frac{n+1}{2}}^{A}
$$


Similarly, since

$$
H_{o}^{D}-H_{e}^{D}=\sum_{i=1}^{\left[\frac{n+1}{4}\right]} \sigma_{\frac{n+1}{2}+2 i-1}^{D}-\sum_{i=1}^{\left[\frac{n-1}{4}\right]} \sigma_{\frac{n+1}{2}+2 i}^{D}
$$

and using the expressions above, we obtain that

$$
H_{o}^{D}-H_{e}^{D}=\frac{\sum_{i=1}^{\left[\frac{n}{2}\right]}(-1)^{i+1} \varphi_{i}}{1-\sum_{i=1}^{\left[\frac{n}{2}\right]}(-1)^{i+1} \psi_{i}} \sigma_{\frac{n+1}{2}}^{D}
$$

Now we can substitute this into the previous expressions for the $\sigma^{D}$ 's and obtain that for $0 \leqslant j<\frac{n+1}{2}$

$$
\sigma_{\frac{n+1}{2}+j}^{D}=\left[\varphi_{j}-\psi_{j} \frac{\sum_{i=1}^{\left[\frac{n}{2}\right]}(-1)^{i} \varphi_{i}}{1+\sum_{i=1}^{\left[\frac{n}{2}\right]}(-1)^{i} \psi_{i}}\right] \sigma_{\frac{n+1}{2}}^{D}
$$

Since $\sigma^{A}$ and $\sigma^{D}$ are probability distributions we must have

$$
\begin{aligned}
\sigma_{\frac{n+1}{2}}^{A}+2 \sum_{i=1}^{\left[\frac{n}{2}\right]} \sigma_{i}^{D A} & =1 \\
\sigma_{\frac{n+1}{2}}^{D}+2 \sum_{i=1}^{\left[\frac{n}{2}\right]} \sigma_{i}^{D} & =1 \\
\sigma_{k}^{A} & \geq 0, \quad k=1,2, \ldots, n \\
\sigma_{k}^{D} & \geq 0, \quad k=1,2, \ldots, n
\end{aligned}
$$

As in the even case we denote by $k^{*}=\frac{n+1}{2}-j^{*}$ the extreme of the support for $\sigma^{A}$, that is the first strategy with positive probability. $j^{*}$ is determined by: 


$$
j^{*}=\max \left\{j \geqslant 1: \phi_{j} \geq \beta_{j} \frac{\sum_{i=1}^{j}(-1)^{i} \phi_{i}}{1+\sum_{i=1}^{j}(-1)^{i} \beta_{i}}\right\}
$$

Therefore, we can solve the two recursive systems explicitly to obtain the following probabilities: for candidate $A$ :

$$
\begin{aligned}
& \sigma_{\frac{n+1}{2}}^{A}=\frac{1}{1+2 \sum_{j=1}^{j^{*}}\left[\phi_{j}-\beta_{j} \frac{\sum_{i=1}^{j^{*}}(-1)^{i} \phi_{i}}{1+\sum_{i=1}^{j^{*}}(-1)^{i} \beta_{i}}\right]} \\
& \sigma_{\frac{n+1}{2}+j}^{A}=\left[\phi_{j}-\beta_{j} \frac{\sum_{i=1}^{j^{*}}(-1)^{i} \phi_{i}}{1+\sum_{i=1}^{j^{*}}(-1)^{i} \beta_{i}}\right] \sigma_{\frac{n+1}{2}}^{A} \quad \text { for } 1 \leq j \leq j^{*}
\end{aligned}
$$

and, since the limits of the support of candidate $D$ are determined by the $k^{*}$ found above, we have that for candidate $D$ :

$$
\begin{gathered}
\sigma_{\frac{n+1}{2}}^{D}=\frac{1}{1+2 \sum_{j=1}^{j^{*}}\left[\varphi_{j}-\psi_{j} \frac{\sum_{i=1}^{j^{*}}(-1)^{i} \varphi_{i}}{1+\sum_{i=1}^{j^{*}}(-1)^{i} \psi_{i}}\right]} \\
\sigma_{\frac{n+1}{2}+j}^{D}=\left[\varphi_{j}-\psi_{j} \frac{\sum_{i=1}^{j^{*}}(-1)^{i} \varphi_{i}}{1+\sum_{i=1}^{j^{*}}(-1)^{i} \psi_{i}}\right] \sigma_{\frac{n+1}{2}}^{D} \text { for } 1 \leq j \leq j^{*}
\end{gathered}
$$

or 


$$
\sigma_{\frac{n+1}{2}+j}^{D}=\left[\varphi_{j}-\psi_{j} \frac{\sum_{i=1}^{j^{*}+1}(-1)^{i} \varphi_{i}}{1+\sum_{i=1}^{j^{*}+1}(-1)^{i} \psi_{i}}\right] \sigma_{\frac{n+1}{2}}^{D} \text { for } 1 \leq j \leq j^{*}+1
$$

\subsection{Equilibrium in symmetric strategies}

Proposition 6: In any equilibrium with symmetric strategies and no gaps,

if $k \leqslant \frac{n+1}{3}$ then $\sigma_{k}^{D} \geqslant \sigma_{k+1}^{D}$

if $k \leqslant \frac{n+1}{2}$ then $\sigma_{k}^{D} \geqslant \sigma_{k+2}^{D}$

and

if $k \geq \frac{2(n+1)}{3}$ then $\sigma_{k}^{D} \leq \sigma_{k+1}^{D}$

if $k \geq \frac{n+1}{2}$ then $\sigma_{k}^{D} \leq \sigma_{k+2}^{D}$

\section{Proof:}

If we equate the payoffs that candidate $A$ obtains from strategies $k$ and $k+2$ we obtain the following set of equations:

$$
k \sigma_{k}^{D}+\sum_{i=1}^{k-1} \sigma_{k-i}^{D}=(n-2 k-1) \sigma_{k+1}^{D}+(n-k-1) \sigma_{k+2}^{D}+\sum_{i=3}^{n-k} \sigma_{k+i}^{D}
$$

for $1 \leqslant k \leqslant n-2$

and if we assume symmetry we can it write it as:

$$
(k-1) \sigma_{k}^{D}=(n-2 k) \sigma_{k+1}^{D}+(n-k) \sigma_{k+2}^{D}+2 \sum_{i=3}^{\frac{n-1}{2}} \sigma_{k+i}^{D}+\sigma_{\frac{n+1}{2}}^{D}
$$

for $1 \leqslant k<\frac{n+1}{2}-1$. And for $k=\frac{n+1}{2}$ we have

$$
\left(\frac{n+1}{2}\right) \sigma_{\frac{n+1}{2}}^{D}+3 \sigma_{\frac{n+1}{2}}^{D}=\left(\frac{n+1}{2}-3\right) \sigma_{\frac{n+1}{2}+2}^{D}
$$

For $k=\frac{n+1}{2}-1$ we obtain an identity.

If $k \leqslant \frac{n+1}{2}$ then $n-k \geqslant k-1$. From the above equation, this immediately implies that $\sigma_{k}^{D} \geqslant \sigma_{k+2}^{D}$. Similarly if $k \leqslant \frac{n+1}{3}$ then $n-2 k \geqslant k-1$. From the above equation, 
this immediately implies that $\sigma_{k}^{D} \geqslant \sigma_{k+1}^{D}$. The other two results can be obtained by symmetry.

Proposition 7: In any equilibrium with symmetric strategies and no gaps,

if $k<\frac{n+1}{3}$ then $\sigma_{k}^{A} \leqslant \sigma_{k+1}^{A}$

if $k<\frac{n+1}{2}$ then $\sigma_{k}^{A} \leqslant \sigma_{k+2}^{A}$

and

if $k>\frac{2(n+1)}{3}$ then $\sigma_{k}^{A} \geq \sigma_{k+1}^{A}$

if $k>\frac{n+1}{2}$ then $\sigma_{k}^{A} \geq \sigma_{k+2}^{A}$

Proof:

If we equate the payoffs that candidate $D$ obtains from strategies $k$ and $k+2$ we obtain the following set of equations:

$$
\begin{aligned}
\ldots+\sigma_{k-2}^{A}+\sigma_{k-1}^{A}+k \sigma_{k+2}^{A} & =(n-k-1) \sigma_{k}^{A}+(n-2 k-1) \sigma_{k+1}^{A}+\sigma_{k+3}^{A}+\sigma_{k+4}^{A}+\ldots \\
k \sigma_{k+2}^{A}+\sum_{i=1}^{k-1} \sigma_{k-i}^{A} & =(n-k-1) \sigma_{k+2}^{A}+(n-2 k-1) \sigma_{k+1}^{A}+\sum_{i=3}^{n-k} \sigma_{k+i}^{A}
\end{aligned}
$$

for $1 \leqslant k \leqslant n-2$

If we assume symmetry we can write them as

$$
k \sigma_{k+2}^{A}=(n-k-1) \sigma_{k}^{A}+(n-2 k-1) \sigma_{k+1}^{A}+2 \sum_{i=3}^{\frac{n-1}{2}} \sigma_{k+i}^{A}+\sigma_{\frac{n+1}{2}}^{A}
$$

for $1 \leqslant k<\frac{n+1}{2}-1$. And for $k=\frac{n+1}{2}$ we have that

$$
\left(\frac{n+1}{2}+1\right) \sigma_{\frac{n+1}{2}+2}^{A}=\left(\frac{n+1}{2}-2\right) \sigma_{\frac{n+1}{2}}^{A}-3 \sigma_{\frac{n+1}{2}+1}^{A}
$$

For $k=\frac{n+1}{2}-1$ we obtain an identity.

If $k<\frac{n+1}{2}$ then $n-k-1 \geqslant k$. From the above equation, this immediately implies that $\sigma_{k}^{A} \leq \sigma_{k+2}^{A}$. Similarly if $k<\frac{n+1}{3}$ then $n-2 k-1 \geqslant k$. From the above equation, this immediately implies that $\sigma_{k}^{A} \leq \sigma_{k+1}^{A}$. The other two results can be obtained by symmetry.

That is, the disadvantaged candidate's mixing distribution is almost $U$-shaped, with the least probability weight in the center, and increasing probability in every other strategy as they move away from the center. In contrast, candidate $A$ 's mixing 
distribution places monotonically decreasing weight on every other strategy that is further from the median. Even though in all our examples the disadvantaged candidate's distribution is $U$-shaped, in general it could produce a shape of two parallel $U$-shaped distributions since we can only show that the probabilities decrease every other location when approaching the center of the interval. Similarly, in the examples we find that the distribution of the advantaged candidate is monotone at each side of the center of the interval, but in general we can only prove an approximation of it.

\subsection{Equilibrium for $n \leq 7$}

Since the derivation above only applies to the case of $n>7$ we computed directly the solutions for smaller odd values of $n$. These are given in the table below.

\begin{tabular}{|c|c|c|}
\hline$n$ & $\sigma^{A}$ & $\sigma^{D}$ \\
\hline 3 & $.2, .6, .2$ & $.4, .2, .4$ \\
\hline 5 & $0, .25, .5, .25,0$ & $0, .375, .25, .375,0$ \\
\hline 7 & $0, .02, .27, .42, .27, .02,0$ & $0, .375, .125,0, .125, .375,0$ \\
\hline
\end{tabular}

\subsection{Convergence to the even case}

We will show that as $n$ becomes large, the mixing distribution found in the last section approach the ones found for the case when $n$ is even. We prove two lemmas, and the result follows directly from them.

Lemma 1: $\lim _{n \rightarrow \infty} \beta_{j}=0$ and $\lim _{n \rightarrow \infty} \psi_{j}=0$

Proof:

We will show it by induction. Since $\beta_{0}=0$ and $\beta_{1}=\frac{-1}{\frac{n+1}{2}}$, we have that $\lim _{n \rightarrow \infty} \beta_{1}=0$. Now suppose that $\lim _{n \rightarrow \infty} \beta_{j}=0$ for all $j<j^{\prime}$. In that case we also have that and for

$$
\lim _{n \rightarrow \infty} \beta_{j^{\prime}}=\lim _{n \rightarrow \infty} \frac{\left(\frac{n+1}{2}-j^{\prime}-1\right) \beta_{j^{\prime}-1}+(-1)^{j^{\prime}}-\sum_{i=1}^{j^{\prime}-2} \beta_{i}}{\frac{n+1}{2}+j^{\prime}-1}=0
$$

Similarly, we have that $\psi_{0}=0$ and $\psi_{1}=-\frac{1}{\frac{n+1}{2}-2}$. Therefore, $\lim _{n \rightarrow \infty} \psi_{1}=0$. Now suppose that $\lim _{n \rightarrow \infty} \psi_{j}=0$ for all $j<j^{\prime}$. In that case we also have that 


$$
\lim _{n \rightarrow \infty} \psi_{j}=\lim _{n \rightarrow \infty} \frac{\left(\frac{n+1}{2}+j-1\right) \psi_{j-1}+(-1)^{j}+\sum_{i=1}^{j-2} \psi_{i}}{\frac{n+1}{2}-j-1}=0
$$

Thus, the role of $\left(H_{o}^{A}-H_{e}^{A}\right)$ and $\left(H_{o}^{D}-H_{e}^{D}\right)$ in determining the probabilities $\sigma^{A}$ and $\sigma^{D}$ respectively, decreases as $n$ gets large. On the other hand:

Lemma 2: $\lim _{n \rightarrow \infty} \phi_{j}=1$ and $\lim _{n \rightarrow \infty} \varphi_{j}=1$

Proof:

We will show it by induction. Since $\phi_{0}=1$ and $\phi_{1}=\frac{\frac{n+1}{2}-1}{\frac{n+1}{2}}$, we have that $\lim _{n \rightarrow \infty} \phi_{1}=1$. Now suppose that $\lim _{n \rightarrow \infty} \phi_{j}=1$ for all $j<j^{\prime}$. In that case we also have that and for

$$
\lim _{n \rightarrow \infty} \phi_{j^{\prime}}=\lim _{n \rightarrow \infty} \frac{\left(\frac{n+1}{2}-j^{\prime}-1\right) \phi_{j^{\prime}-1}-\sum_{i=0}^{j^{\prime}-2} \phi_{i}}{\frac{n+1}{2}+j^{\prime}-1}=1 \text { if } j^{\prime} \text { is even }
$$

and

$$
\lim _{n \rightarrow \infty} \phi_{j^{\prime}}=\lim _{n \rightarrow \infty} \frac{\left(\frac{n+1}{2}-j^{\prime}-1\right) \phi_{j^{\prime}-1}-\sum_{i=1}^{j^{\prime}-2} \phi_{i}}{\frac{n+1}{2}+j^{\prime}-1}=1 \text { if } j^{\prime} \text { is odd }
$$

Similarly, we have that $\varphi_{0}=1$ and $\varphi_{1}=\frac{\frac{n+1}{2}}{\frac{n+1}{2}-2}$. Therefore, $\lim _{n \rightarrow \infty} \varphi_{1}=1$. Now suppose that $\lim _{n \rightarrow \infty} \varphi_{j}=1$ for all $j<j^{\prime}$. In that case we also have that

$$
\lim _{n \rightarrow \infty} \varphi_{j^{\prime}}=\lim _{n \rightarrow \infty} \frac{\left(\frac{n+1}{2}+j^{\prime}-1\right) \varphi_{j^{\prime}-1}+\sum_{i=0}^{j^{\prime}-2} \varphi_{i}}{\frac{n+1}{2}-j^{\prime}-1}=1 \text { if } j^{\prime} \text { is odd }
$$

and

$$
\lim _{n \rightarrow \infty} \varphi_{j^{\prime}}=\lim _{n \rightarrow \infty} \frac{\left(\frac{n+1}{2}+j^{\prime}-1\right) \varphi_{j^{\prime}-1}+\sum_{i=1}^{j^{\prime}-2} \varphi_{i}}{\frac{n+1}{2}-j^{\prime}-1}=1 \text { if } j^{\prime} \text { is even }
$$


Suppose that $n$ is even and consider the equilibrium strategies corresponding to $\sigma^{A}(n), \sigma^{D}(n), \sigma^{A}(n+1)$ and $\sigma^{D}(n+1)$. Given the previous results we have that

$$
\lim _{n \rightarrow \infty} \sigma^{A}(n)-\sigma^{A}(n+1)=\lim _{n \rightarrow \infty} \sigma^{D}(n)-\sigma^{D}(n+1)=0
$$

since

$$
\lim _{n \rightarrow \infty} \frac{\sigma_{\frac{n}{2}}^{A}(n)-\sigma_{\frac{n+2}{2}}^{A}(n+1)=}{1+2\left(\sum_{1 \leq j \leq j_{A}^{*}} S_{j}^{A}(n)\right)}-\frac{1}{1+2 \sum_{j=1}^{j^{*}}\left[\phi_{j}(n+1)-\beta_{j}(n+1) \frac{\sum_{i=1}^{j^{*}(-1)^{i} \phi_{i}(n+1)}}{1+\sum_{i=1}^{j^{*}}(-1)^{i} \beta_{i}(n+1)}\right]}=0
$$

and

$$
\lim _{n \rightarrow \infty} \frac{\sigma_{\frac{n}{2}}^{D}(n)-\sigma_{\frac{n+2}{2}}^{D}(n+1)=}{1+2\left(\sum_{1 \leq i \leq j_{D}^{*}} S_{i}^{D}(n)\right)}-\frac{1}{1+2 \sum_{j=1}^{j^{*}}\left[\varphi_{j}(n+1)-\psi_{j}(n+1) \frac{\sum_{i=1}^{j^{*}}(-1)^{i} \varphi_{i}(n+1)}{1+\sum_{i=1}^{j^{*}}(-1)^{i} \psi_{i}(n+1)}\right]}=0
$$

Since for all $n$ we have that

$$
\begin{aligned}
\sigma_{\frac{n+1}{2}+j}^{A} & =\phi_{j}(n) \sigma_{\frac{n+1}{2}}^{A}+\beta_{j}(n)\left(H_{o}^{A}(n)-H_{e}^{A}(n)\right) \\
\sigma_{\frac{n+1}{2}+j}^{D} & =\varphi_{j}(n) \sigma_{\frac{n+1}{2}}^{D}+\psi_{j}(n)\left(H_{o}^{D}(n)-H_{e}^{D}(n)\right)
\end{aligned}
$$

and

$$
\begin{aligned}
\sigma_{\frac{n}{2}-j}^{A} & =S_{j}^{A}(n) \sigma_{\frac{n}{2}}^{A} \\
\sigma_{\frac{n}{2}-j}^{D} & =S_{j}^{D}(n) \sigma_{\frac{n}{2}}^{A}
\end{aligned}
$$

we also have that

$$
\begin{aligned}
& \lim _{n \rightarrow \infty} \sigma_{\frac{n}{2}+j}^{A}-\sigma_{\frac{n+2}{2}+j}^{A}= \\
& \lim _{n \rightarrow \infty} S_{j}^{A}(n) \sigma_{\frac{n}{2}}^{A}-\phi_{j}(n+1) \sigma_{\frac{n+2}{2}}^{A}-\beta_{j}(n+1)\left(H_{o}^{A}(n+1)-H_{e}^{A}(n+1)\right)=0 \\
& \text { and } \\
& \lim _{n \rightarrow \infty} \sigma_{\frac{n}{2}+j}^{D}-\sigma_{\frac{n+2}{2}+j}^{D}= \\
& \lim _{n \rightarrow \infty} S_{j}^{D}(n) \sigma_{\frac{n}{2}}^{D}-\varphi_{j}(n+1) \sigma_{\frac{n+2}{2}}^{D}-\psi_{j}(n+1)\left(H_{o}^{D}(n+1)-H_{e}^{D}(n+1)\right)=0
\end{aligned}
$$

Therefore the equilibrium probabilities $\sigma^{A}$ and $\sigma^{D}$ found for the case when $n$ is odd approach the values found for the case when $n$ is even. 


\section{Appendix B: Proof of Theorem 5: Existence of equilibrium with continuous locations.}

First, we observe that if $\delta \geq \frac{1}{2}$ then there are many pure strategy equilibria, where $A$ locates sufficiently close to $\frac{1}{2}, D$ locates anywhere, and $A$ wins with probability 1 . When $\delta<\frac{1}{2}$ it is straightforward to show that no pure strategy equilibrium exists, since, for every location $A$ chooses, there exists a response by $D$ than gives $D$ a strictly positive payoff, but for any location $D$ might choose, $A$ can match $D$, which leaves $D$ with a 0 payoff. Hence there is no minimax point in pure strategies if $\delta<\frac{1}{2}$.

The main theorem of Dasgupta and Maskin (1986, p.14) states:

Let $\left[\left(S_{i}, U_{i}\right) ; i=1, \ldots, N\right]$ be a game. Let $S_{i} \subseteq R^{1}(i=1, \ldots, N)$ be a closed interval and let $U_{i}: S \rightarrow R^{1}(i=1, \ldots, N)$ be continuous except on a subset $S^{* *}(i)$ of $S^{*}(i)$, where $S^{*}(i)$ is defined by

$$
S^{*}(i)=\left\{\left(s_{1}, \ldots, s_{N}\right) \in S: \exists j \neq i, \exists d, 1 \leq d \leq \Delta(i) \text { such that } s_{j}=f_{i j}^{d}\left(s_{i}\right)\right\}
$$

where $\Delta(i)$ is a positive integer and for each integer $d$, with $1 \leq d \leq \Delta(i)$, and $f_{i j}^{d}: R^{1} \rightarrow R^{1}$ is a one-to-one, continuous function. Suppose $\sum_{i=1}^{N} U_{i}(\mathbf{s})$ is upper semi-continuous and $U_{i}\left(s_{i}, \mathbf{s}_{-i}\right)$ is bounded and weakly lower semi-continuous in $s_{i}$. Then the game $\left[\left(S_{i}, U_{i}\right) ; i=1, \ldots, N\right]$ possesses a mixed-strategy equilibrium.

To prove Theorem 5 for the case of $\delta<\frac{1}{2}$ we show that the conditions of the Dasgupta-Maskin theorem are satisfied. When we apply this theorem to our case we have:

1. $S_{i}=\wp=[0,1]$ for $i=A, D$, is a closed interval.

2. $\pi_{A}\left(x_{A}, x_{B}\right)$ and $\pi_{B}\left(x_{A}, x_{B}\right)$ are continuous except on a set of measure zero. We may take $\Delta(A)=\Delta(D)=2, f_{A, D}^{1}\left(x_{A}\right)=x_{A}-\delta$, and $f_{A, D}^{2}\left(x_{A}\right)=x_{A}+\delta$. In our case $S^{*}(A)=S^{*}(D)$, the union of two 45 degree lines.

3. $\pi_{A}\left(x_{A}, x_{D}\right)+\pi_{D}\left(x_{D}, x_{A}\right)=1$, which is a constant function so $\sum_{i=1}^{N} U_{i}(\mathbf{s})$ is upper semi-continuous.

4. $0 \leq \pi_{i}\left(x_{A}, x_{D}\right) \leq 1$ and therefore $U_{i}\left(s_{i}, \mathbf{s}_{-i}\right)$ is bounded for each player

5. The proof that $\pi_{i}\left(x_{A}, x_{D}\right)$ is weakly lower semi-continuous in $x_{i}$ for $i=A, D$ requires several few steps, which are given below. The definition of weak lower semi-continuity is: 
Definition 3: $U_{i}\left(s_{i}, \mathbf{s}_{-i}\right)$ is weakly lower semi-continuous in $s_{i}$ if $\forall \bar{s}_{i} \in S_{i}^{* *}(i)$, $\exists \lambda \in[0,1]$ such that $\forall s_{-i} \in S_{-i}^{* *}\left(\bar{s}_{i}\right)$,

$\lambda \liminf _{s_{i} \rightarrow-\bar{s}_{i}} U_{i}\left(s_{i}, \mathbf{s}_{-i}\right)+(1-\lambda) \liminf _{s_{i} \rightarrow+\bar{s}_{i}} U_{i}\left(s_{i}, \mathbf{s}_{-i}\right) \geq U_{i}\left(\bar{s}_{i}, \mathbf{s}_{-i}\right)$.

First consider $\pi_{A}\left(x_{A}, x_{D}\right)$, and let $x_{D}$ be fixed. We treat five separate cases:

Case $1 \mathrm{~A} x_{D} \in(\delta, 1-\delta)$. In this case there are two discontinuities, one at $x_{A}=x_{D}-\delta$ and the other at $x_{A}=x_{D}+\delta$. For any $x_{A}$ strictly between these two discontinuity points, $\pi_{A}\left(x_{A}, x_{D}\right)=1$. At the first discontinuity point, $\pi_{A}\left(x_{D}-\delta, x_{D}\right)=$ $\frac{1+x_{D}}{2}, \liminf _{x_{A} \rightarrow-x_{D}-\delta} \pi_{A}\left(x_{A}, x_{D}\right)=x_{D}$, and $\liminf _{x_{A} \rightarrow^{+} x_{D}-\delta} \pi_{A}\left(x_{A}, x_{D}\right)=1$. Thus we can take any $\lambda \leq \frac{1}{2}$ and satisfy the required inequality:

$$
\lambda x_{D}+(1-\lambda) \cdot 1 \geq \frac{1+x_{D}}{2}
$$

Similarly, at the second discontinuity point, $\pi_{A}\left(x_{D}+\delta, x_{D}\right)=\frac{2-x_{D}}{2}, \liminf _{x_{A} \rightarrow^{-} x_{D}+\delta} \pi_{A}\left(x_{A}, x_{D}\right)=$ 1 , and $\liminf _{x_{A} \rightarrow x_{D}+\delta} \pi_{A}\left(x_{A}, x_{D}\right)=\left(1-x_{D}\right)$. Thus we can take any $\lambda \geq \frac{1}{2}$ and satisfy the required inequality:

$$
\lambda \cdot 1+(1-\lambda)\left(1-x_{D}\right) \geq \frac{2-x_{D}}{2}
$$

Case 2A $x_{D} \in(0, \delta)$. In this case there is one discontinuity, at $x_{A}=x_{D}+\delta$. For any $x_{A}<x_{D}+\delta, \pi_{A}\left(x_{A}, x_{D}\right)=1$. At the discontinuity point, $\pi_{A}\left(x_{D}+\delta, x_{D}\right)=$ $\frac{2-x_{D}}{2}, \liminf x_{x_{A} \rightarrow^{-} x_{D}+\delta} \pi_{A}\left(x_{A}, x_{D}\right)=1$, and $\liminf _{x_{A} \rightarrow^{+} x_{D}+\delta} \pi_{A}\left(x_{A}, x_{D}\right)=\left(1-x_{D}\right)$.

Thus we can take any $\lambda \leq \frac{1}{2}$ and satisfy the required inequality:

$$
\lambda \cdot 1+(1-\lambda)\left(1-x_{D}\right) \geq \frac{2-x_{D}}{2}
$$

Case 3A $x_{D} \in(1-\delta, 1)$. In this case there is one discontinuity, at $x_{A}=x_{D}-\delta$. For any $x_{A}>x_{D}-\delta, \pi_{A}\left(x_{A}, x_{D}\right)=1$. At the discontinuity point, $\pi_{A}\left(x_{D}+\delta, x_{D}\right)=$ $\frac{1+x_{D}}{2}, \liminf _{x_{A} \rightarrow^{-} x_{D}-\delta} \pi_{A}\left(x_{A}, x_{D}\right)=x_{D}$, and $\liminf _{x_{A} \rightarrow^{+} x_{D}-\delta} \pi_{A}\left(x_{A}, x_{D}\right)=1$. Thus we can take any $\lambda \geq \frac{1}{2}$ and satisfy the required inequality:

$$
\lambda x_{D}+\lambda \cdot 1 \geq \frac{1+x_{D}}{2}
$$

Case 4A $x_{D}=1-\delta$. In this case there are two discontinuities, with one at $x_{A}=$ $x_{D}-\delta$ and the other at the right boundary, $x_{A}=1$. The first discontinuity can 
be dealt with as in Case 3. For the second discontinuity, the definition of weak lower semi-continuity requires that $\liminf _{s_{i} \rightarrow-\bar{s}_{i}} U_{i}\left(s_{i}, \mathbf{s}_{-i}\right) \geq U_{i}\left(\bar{s}_{i}, \mathbf{s}_{-i}\right)$. This is satisfied since $\liminf x_{x_{A} \rightarrow^{-1}} \pi_{A}\left(x_{A}, 1-\delta\right)=1$ and $\pi_{A}(1,1-\delta)=\frac{1+\delta}{2} \leq 1$.

Case 5A $x_{D}=\delta$. In this case there are two discontinuities, with one at $x_{A}=x_{D}+\delta$ and the other at the left boundary, $x_{A}=0$. The first discontinuity can be dealt with as in Case 2. For the second discontinuity, the definition of weak lower semi-continuity requires that $\liminf _{s_{i} \rightarrow+\bar{s}_{i}} U_{i}\left(s_{i}, \mathbf{s}_{-i}\right) \geq U_{i}\left(\bar{s}_{i}, \mathbf{s}_{-i}\right)$. This is satisfied since $\liminf x_{x_{A} \rightarrow+} \pi_{A}\left(x_{A}, \delta\right)=1$ and $\pi_{A}(0, \delta)=\frac{1+\delta}{2} \leq 1$.

Now consider $\pi_{D}\left(x_{D}, x_{A}\right)$, and let $x_{A}$ be fixed. Again, we treat five separate cases:

Case 1D $x_{A} \in(\delta, 1-\delta)$. In this case there are two discontinuities, one at $x_{D}=x_{A}-\delta$ and the other at $x_{D}=x_{A}+\delta$. For any $x_{D}$ strictly between these two discontinuity points, $\pi_{D}\left(x_{A}, x_{D}\right)=0$. At the first discontinuity point, $\pi_{D}\left(x_{A}, x_{A}-\delta\right)=$ $\frac{x_{A}-\delta}{2}, \liminf _{x_{D} \rightarrow^{-} x_{A}-\delta} \pi_{D}\left(x_{A}, x_{D}\right)=x_{A}-\delta,{\text { and } \liminf _{x_{D} \rightarrow+} x_{A}-\delta} \pi_{D}\left(x_{A}, x_{D}\right)=$ 0 . Thus we can take any $\lambda \geq \frac{1}{2}$ and satisfy the required inequality:

$$
\lambda\left(x_{A}-\delta\right)+(1-\lambda) \cdot 0 \geq \frac{x_{A}-\delta}{2}
$$

Similarly, at the second discontinuity point, $\pi_{D}\left(x_{A}, x_{A}+\delta\right)=\frac{1-\left(x_{A}+\delta\right)}{2}$, $\liminf _{x_{D} \rightarrow-x_{A}+\delta} \pi_{D}\left(x_{A}, x_{D}\right)=0$, and $\liminf _{x_{D} \rightarrow+x_{A}-\delta} \pi_{D}\left(x_{A}, x_{D}\right)=1-\left(x_{A}+\right.$ $\delta)$. Thus we can take any $\lambda \leq \frac{1}{2}$ and satisfy the required inequality:

$$
\lambda \cdot 0+(1-\lambda)\left(1-\left(x_{A}+\delta\right)\right) \geq \frac{1-\left(x_{A}+\delta\right)}{2}
$$

Case 2D $x_{A} \in(0, \delta)$. In this case there is one discontinuity, at $x_{D}=x_{A}+\delta$. For any $x_{D}<x_{A}+\delta, \pi_{D}\left(x_{A}, x_{D}\right)=0$. At the discontinuity point, $\pi_{D}\left(x_{A}, x_{A}+\delta\right)=$ $\frac{1-\left(x_{A}+\delta\right)}{2}, \liminf _{x_{D} \rightarrow{ }^{-} x_{A}+\delta} \pi_{D}\left(x_{A}, x_{D}\right)=0$, and $\liminf _{x_{D} \rightarrow{ }^{+} x_{A}+\delta} \pi_{D}\left(x_{A}, x_{D}\right)=$ $1-\left(x_{A}+\delta\right)$. Thus we can take any $\lambda \leq \frac{1}{2}$ and satisfy the required inequality:

$$
\lambda \cdot 0+(1-\lambda)\left(1-\left(x_{A}+\delta\right)\right) \geq \frac{1-\left(x_{A}+\delta\right)}{2}
$$

Case 3D $x_{A} \in(1-\delta, 1)$. In this case there is one discontinuity, at $x_{D}=x_{A}-\delta$. For any $x_{D}>x_{A}-\delta, \pi_{D}\left(x_{A}, x_{D}\right)=0$. At the discontinuity point, $\pi_{D}\left(x_{A}, x_{A}+\delta\right)=$ 
$\frac{x_{A}-\delta}{2}, \liminf _{x_{D} \rightarrow^{-} x_{A}+\delta} \pi_{D}\left(x_{A}, x_{D}\right)=x_{A}-\delta, \operatorname{and~} \liminf _{x_{D} \rightarrow{ }^{+} x_{A}+\delta} \pi_{D}\left(x_{A}, x_{D}\right)=$ 0 . Thus we can take any $\lambda \geq \frac{1}{2}$ and satisfy the required inequality:

$$
\lambda\left(x_{A}-\delta\right)+(1-\lambda) \cdot 0 \geq \frac{x_{A}-\delta}{2}
$$

Case $4 \mathrm{D} x_{A}=1-\delta$. In this case there are two discontinuities, with one at $x_{D}=$ $x_{A}-\delta$ and the other at the right boundary, $x_{D}=1$. The first discontinuity can be dealt with as in Case 3. For the second discontinuity, the definition of weak lower semi-continuity requires that $\liminf _{s_{i} \rightarrow^{-} \bar{s}_{i}} U_{i}\left(s_{i}, \mathbf{s}_{-i}\right) \geq U_{i}\left(\bar{s}_{i}, \mathbf{s}_{-i}\right)$. This is satisfied since $\liminf _{x_{D} \rightarrow^{-1}} \pi_{D}\left(1-\delta, x_{D}\right)=0$ and $\pi_{A}(1-\delta, 1)=0 \leq 0$.

Case 5D $x_{A}=\delta$. In this case there are two discontinuities, with one at $x_{D}=x_{A}+\delta$ and the other at the left boundary, $x_{D}=0$. The first discontinuity can be dealt with as in Case 2. For the second discontinuity, the definition of weak lower semi-continuity requires that $\liminf _{s_{i} \rightarrow+\bar{s}_{i}} U_{i}\left(s_{i}, \mathbf{s}_{-i}\right) \geq U_{i}\left(\bar{s}_{i}, \mathbf{s}_{-i}\right)$. This is satisfied since $\liminf _{x_{A} \rightarrow+} \pi_{D}\left(\delta, x_{D}\right)=0$ and $\pi_{A}(\delta, 0)=0 \leq 0$.

Thus, $\pi_{i}\left(x_{A}, x_{D}\right)$ is weakly lower semi-continuous in $x_{i}$ for $i=A, D$. Therefore, our game possesses a mixed-strategy equilibrium. 